\title{
Iterative methods for triple hierarchical variational inequalities and common fixed point problems
}

\author{
DR Sahu', Shin Min Kang ${ }^{2 *}$, Vidya Sagar ${ }^{1}$ and Satyendra Kumar ${ }^{1}$
}

\section{"Correspondence:} smkang@gnu.ac.kr

${ }^{2}$ Department of Mathematics and RINS, Gyeongsang National University, Jinju, 660-701, Korea Full list of author information is available at the end of the article

\begin{abstract}
The purpose of this paper is to introduce a new iterative scheme for approximating the solution of a triple hierarchical variational inequality problem. Under some requirements on parameters, we study the convergence analysis of the proposed iterative scheme for the considered triple hierarchical variational inequality problem which is defined over the set of solutions of a variational inequality problem defined over the intersection of the set of common fixed points of a sequence of nearly nonexpansive mappings and the set of solutions of the classical variational inequality. Our strong convergence theorems extend and improve some known corresponding results in the contemporary literature for a wider class of nonexpansive type mappings in Hilbert spaces.
\end{abstract}

MSC: 47J20; 47J25

Keywords: metric projection mapping; nonexpansive mapping; sequence of nearly nonexpansive mappings; triple hierarchical variational inequality

\section{Introduction}

The classical variational inequality problem initially studied by Stampacchia [1] for a nonlinear operator $A: C \rightarrow H$ is a problem which provides us such $x^{*} \in D$ which satisfies

$$
\left\langle A x^{*}, y-x^{*}\right\rangle \geq 0, \quad \forall y \in D,
$$

where $C$ is a nonempty closed convex subset of a real Hilbert space $H$ and $D$ is a nonempty closed convex subset of $C$. The variational inequality (1.1) is denoted by $\mathrm{VI}_{D}(C, A)$. The set of solutions of (1.1) is denoted by $\Omega_{D}(C, A)$, that is,

$$
\Omega_{D}(C, A)=\left\{x^{*} \in D:\left\langle A x^{*}, y-x^{*}\right\rangle \geq 0, \forall y \in D\right\} .
$$

For $C=D$, we use $\operatorname{VI}(C, A):=\operatorname{VI}_{D}(C, A)$ and $\Omega(C, A):=\Omega_{D}(C, A)$.

In the framework of variational inequality problems, various problems arising in several branches of pure and applied sciences can be studied (see [2, 3]).

The equivalence relation between the variational inequality and fixed point problems can be seen by projection technique which plays an important role in developing an important role in developing some efficient methods for solving variational inequality problems

\section{基 Springer}

๑2014 Sahu et al.; licensee Springer. This is an Open Access article distributed under the terms of the Creative Commons Attribution License (http://creativecommons.org/licenses/by/2.0), which permits unrestricted use, distribution, and reproduction in any medium, provided the original work is properly cited. 
and related optimization problems. The problem of finding the fixed points of a nonexpansive mapping is the subject of current interest related to variational inequality problems in functional analysis.

Over the set of fixed points of a nonexpansive mapping, several authors (see [4-7] etc.) have studied the variational inequality problem in a particular manner. This kind of variational inequality is named a hierarchical variational inequality; it is defined as follows:

Find $x^{*} \in F(S)$ such that $\left\langle(I-T) x^{*}, y-x^{*}\right\rangle \geq 0, \quad \forall y \in F(S)$,

where $T$ and $S$ are two nonexpansive mappings from a nonempty closed convex subset $C$ of a real Hilbert space $H$ into itself, and $F(S)$ denotes the set of fixed points of the mapping $S$. One can easily observe that $\mathrm{VI}_{F(S)}(C, I-T)$ is equivalent to the fixed point problem $x^{*}=P_{F(S)}\left(T x^{*}\right)$, that is, $x^{*}$ is a fixed point of the nonexpansive mapping $P_{F(S)}(T)$, where $P_{F(S)}$ is the metric projection from $H$ onto a nonempty closed convex subset $F(S)$ of $H$.

After all, in the scenario of variational inequality problem, we eagerly discuss such kind of variational inequality problem which is defined over the set of solutions of a variational inequality and the set of fixed points of a nonexpansive mapping, having a triple structure in contrast with bilevel programming problems or hierarchical constrained optimization problems or hierarchical fixed point problems. This kind of variational inequality is called the triple hierarchical variational inequality (see $[8,9])$, which is also called the triple hierarchical constrained optimization problem (see [8]), and it is defined as follows:

$$
\text { Find } x^{*} \in \Omega_{F(S)}(C, A) \text { such that }\left\langle F x^{*}, y-x^{*}\right\rangle \geq 0, \quad \forall y \in \Omega_{F(S)}(C, A) \text {, }
$$

where $\Omega_{F(S)}(C, A)$ is the set of solutions of $\operatorname{VI}_{F(S)}(C, A) \neq \emptyset$, and mappings $A, F$, and $S$ are inverse strongly monotone, strongly monotone and Lipschitz continuous, and nonexpansive from a nonempty closed convex subset $C$ of a real Hilbert space $H$ into itself, respectively. If $\Omega_{F(S)}(C, I-T)$ is nonempty, then the metric projection $P_{\Omega_{F(S)}(C, I-T)}$ is well defined. The minimum norm solution $x^{*}$ of $\mathrm{VI}_{F(S)}(C, I-T)$ exists uniquely and is exactly the nearest point projection of the origin to $\Omega_{F(S)}(C, I-T)$, that is, $x^{*}=P_{\Omega_{F(S)}(C, I-T)}(0)$. Alternatively, $x^{*}$ is the unique solution of the quadratic minimization problem:

$$
\left\|x^{*}\right\|^{2}=\min \left\{\|x\|^{2}: x \in \Omega_{F(S)}(C, I-T)\right\} .
$$

Finding of this minimum norm solution $x^{*}$ is an interesting problem. In this context, Yao et al. [10] proposed two iterative schemes in an implicit and an explicit both ways to find the minimum norm solution $x^{*}$ of $\mathrm{VI}_{F(S)}(C, I-T)$. They proved two strong convergence results by regularizing the nonexpansive mapping $T$ using contractions.

Recently, Ceng et al. [11], motivated by the results of Yao et al. [10] introduced and studied two iterative schemes, one of which was an implicit while other was an explicit one. They proved two strong convergence results by the considered iterative schemes under suitable conditions on parameters for considered triple hierarchical variational inequalities for both cases. Some hybrid steepest-descent-like methods with variable parameters for triple hierarchical variational inequalities are also studied in Ceng et al. [12]. The importance of the triple hierarchical variational inequalities and a nice survey on this topic is given in [13]. 
In 2005, the first author introduced the class of nearly nonexpansive mappings [14, 15] which is an important generalization of the class of nonexpansive mappings. Let $C$ be a nonempty subset of a Banach space $X$. Fix a sequence $\left\{a_{n}\right\}$ in $[0, \infty)$ with $a_{n} \rightarrow 0$. A mapping $T: C \rightarrow C$ is said to be nearly nonexpansive with respect to the sequence $\left\{a_{n}\right\}$ if for each $n \in \mathbb{N}$,

$$
\left\|T^{n} x-T^{n} y\right\| \leq\|x-y\|+a_{n} \quad \text { for all } x, y \in C .
$$

We now discuss the notion of the sequence of nearly nonexpansive mappings.

Let $C$ be a nonempty subset of a Banach space $X$. Let $\mathcal{T}:=\left\{T_{n}\right\}_{n=1}^{\infty}$ be a sequence of mappings from $C$ into itself. We denote by $F(\mathcal{T})$ the set of common fixed points of the sequence $\mathcal{T}$, that is, $F(\mathcal{T})=\bigcap_{n=1}^{\infty} F\left(T_{n}\right)$. Fix a sequence $\left\{a_{n}\right\}$ in $[0, \infty)$ with $a_{n} \rightarrow 0$, and let $\left\{T_{n}\right\}$ be a sequence of mappings from $C$ into $X$. Then the sequence $\mathcal{T}:=\left\{T_{n}\right\}$ is called a sequence of nearly nonexpansive mappings (see [16]) with respect to a sequence $\left\{a_{n}\right\}$ if

$$
\left\|T_{n} x-T_{n} y\right\| \leq\|x-y\|+a_{n} \quad \text { for all } x, y \in C \text { and } n \in \mathbb{N} .
$$

Clearly, the sequence of nearly nonexpansive mappings can easily be seen to be a wider class of sequence of nonexpansive mappings.

Motivated and inspired by the works mentioned above, we introduce an explicit iterative scheme that generates a sequence and prove that this sequence converges strongly to a unique solution of the considered triple hierarchical variational inequality problem defined over the set of solutions of a variational inequality problem which is defined over the intersection of the set of common fixed points of a sequence of nearly nonexpansive mappings and the set of solutions of the classical variational inequality problem. Our results generalize the result of Ceng et al. [11] in the context of the sequence of nearly nonexpansive mappings and in some other remarkable senses. Our results also extend the result of Yao et al. [10] and many other related works.

\section{Preliminaries}

Throughout this paper, we denote by $\rightarrow$ and $\rightarrow$ the strong convergence and weak convergence, respectively. The symbol $\mathbb{N}$ stands for the set of all natural numbers and $\omega_{w}\left(\left\{x_{n}\right\}\right)$ denotes the set of all weak limits of the sequence $\left\{x_{n}\right\}$.

Let $C$ be a nonempty subset of a real Hilbert space $H$ with inner product $\langle\cdot, \cdot \cdot\rangle$ and norm $\|\cdot\|$, respectively. A mapping $T: C \rightarrow H$ is called

(1) monotone if

$$
\langle T x-T y, x-y\rangle \geq 0 \quad \text { for all } x, y \in C,
$$

(2) $\eta$-strongly monotone if there exists a positive real number $\eta$ such that

$$
\langle T x-T y, x-y\rangle \geq \eta\|x-y\|^{2} \quad \text { for all } x, y \in C,
$$

(3) $\alpha$-inverse strongly monotone if there exists a positive real number $\alpha$ such that

$$
\langle T x-T y, x-y\rangle \geq \alpha\|T x-T y\|^{2} \quad \text { for all } x, y \in C,
$$


(4) $k$-Lipschitzian if there exists a constant $k>0$ such that

$$
\|T x-T y\| \leq k\|x-y\| \quad \text { for all } x, y \in C,
$$

(5) $\rho$-contraction if there exists a constant $\rho \in(0,1)$ such that

$$
\|T x-T y\| \leq \rho\|x-y\| \quad \text { for all } x, y \in C,
$$

(6) nonexpansive if $\|T x-T y\| \leq\|x-y\|$ for all $x, y \in C$,

(7) $\lambda$-strictly pseudocontractive if there exists $\lambda \in[0,1)$ such that

$$
\|T x-T y\|^{2} \leq\|x-y\|^{2}+\lambda\|(I-T) x-(I-T) y\|^{2} \quad \text { for all } x, y \in C,
$$

where $I$ is the identity mapping. Note that if $T: C \rightarrow H$ is $\lambda$-strictly pseudocontractive, then the mapping $A:=I-T$ is $\frac{1-\lambda}{2}$-inverse strongly monotone.

Let $C$ be a nonempty closed convex subset of $H$. Then, for any $x \in H$, there exists a unique nearest point in $C$, denoted by $P_{C}(x)$, such that

$$
\left\|x-P_{C}(x)\right\|=\inf \|x-y\|=: d(x, C) \quad \text { for all } y \in C .
$$

The mapping $P_{C}$ is called the metric projection from $H$ onto $C$ (see Agarwal et al. [14] for some other information related to $P_{C}$ ).

Let $A: C \rightarrow H$ be a monotone and $k$-Lipschitz continuous mapping and let $N_{C}(v)$ be the normal cone to $C$ at $v \in C$, i.e.,

$$
N_{C}(v)=\{w \in H:\langle v-y, w\rangle \geq 0 \text { for all } y \in C\} .
$$

Define

$$
T v= \begin{cases}A v+N_{C}(v), & \text { if } v \in C, \\ \emptyset, & \text { if } v \notin C .\end{cases}
$$

Then $T$ is a maximal monotone and $0 \in T v$ if and only if $v \in \Omega(C, A)$.

Let $C$ be a nonempty subset of a real Hilbert space $H$ and let $T_{1}, T_{2}: C \rightarrow H$ be two mappings. We denote $\mathcal{B}(C)$, the collection of all bounded subsets of $C$. The deviation between $T_{1}$ and $T_{2}$ on $B \in \mathcal{B}(C)$ [16], denoted by $\mathcal{D}_{B}\left(T_{1}, T_{2}\right)$, is defined by

$$
\mathcal{D}_{B}\left(T_{1}, T_{2}\right)=\sup \left\{\left\|T_{1} x-T_{2} x\right\|: x \in B\right\} .
$$

The following lemmas will be needed to prove our main results.

Lemma 2.1 ([17]) The metric projection mapping $P_{C}$ is characterized by the following properties:

(i) $P_{C}(x) \in C$ for all $x \in H$;

(ii) $\left\langle x-P_{C}(x), P_{C}(x)-y\right\rangle \geq 0$ for all $x \in H$ and $y \in C$;

(iii) $\|x-y\|^{2} \geq\left\|x-P_{C}(x)\right\|^{2}+\left\|y-P_{C}(x)\right\|^{2}$ for all $x \in H$ and $y \in C$;

(iv) $\left\langle P_{C}(x)-P_{C}(y), x-y\right\rangle \geq\left\|P_{C}(x)-P_{C}(y)\right\|^{2}$ for all $x, y \in H$. 
Lemma 2.2 ([18]) Let $C$ be a nonempty subset of a real Hilbert space H. Suppose that $\lambda \in(0,1)$ and $\mu>0$. Let $F: C \rightarrow H$ be a k-Lipschitzian and $\eta$-strongly monotone operator on $C$. Define the mapping $W: C \rightarrow H$ by

$$
W x=x-\lambda \mu F(x) \text { for all } x \in C \text {. }
$$

Then $W$ is a contraction provided $\mu<\frac{2 \eta}{k^{2}}$. More precisely, for $\mu \in\left(0, \frac{2 \eta}{k^{2}}\right)$,

$$
\|W x-W y\| \leq(1-\lambda \tau)\|x-y\| \quad \text { for all } x, y \in C,
$$

where $\tau=1-\sqrt{1-\mu\left(2 \eta-\mu k^{2}\right)} \in(0,1]$.

Lemma 2.3 ([14]) Let $T$ be a nonexpansive self-mapping of a nonempty closed convex subset $C$ of a real Hilbert space H. Then $I-T$ is demiclosed at zero, i.e., if $\left\{x_{n}\right\}$ is a sequence in $C$ weakly converging to some $x \in C$ and the sequence $\left\{(I-T) x_{n}\right\}$ strongly converges to 0 , then $x \in F(T)$.

Lemma 2.4 ([19]) Assume $\left\{s_{n}\right\}$ is a sequence of nonnegative real numbers such that

$$
s_{n+1} \leq\left(1-\alpha_{n}\right) s_{n}+\alpha_{n} \beta_{n} \quad \text { for all } n \in \mathbb{N},
$$

where $\left\{\alpha_{n}\right\}$ and $\left\{\beta_{n}\right\}$ are sequences of nonnegative real numbers which satisfy the conditions:

(i) $\left\{\alpha_{n}\right\}_{n=1}^{\infty} \subset(0,1)$ and $\sum_{n=1}^{\infty} \alpha_{n}=\infty$;

(ii) $\lim \sup _{n \rightarrow \infty} \beta_{n} \leq 0$, or

(ii) ${ }^{\prime} \sum_{n=1}^{\infty} \alpha_{n} \beta_{n}$ is convergent.

Then $\lim _{n \rightarrow \infty} s_{n}=0$.

Lemma 2.5 ([20]) Let $C$ be a nonempty closed convex subset of a real Hilbert space $H$ and let $\lambda_{i}>0(i=1,2,3, \ldots, N)$ such that $\sum_{i=1}^{N} \lambda_{i}=1$. Let $T_{1}, T_{2}, T_{3}, \ldots, T_{N}: C \rightarrow C$ be nonexpansive mappings with $\bigcap_{i=1}^{N} F\left(T_{i}\right) \neq \emptyset$ and let $T=\sum_{i=1}^{N} \lambda_{i} T_{i}$. Then $T$ is nonexpansive from $C$ into itself and $F(T)=\bigcap_{i=1}^{N} F\left(T_{i}\right)$.

Proposition 2.1 ([21]) Let C be a nonempty subset of a real Hilbert space H. Let $A: C \rightarrow H$ be an $\alpha$-inverse strongly monotone mapping. Then, the mapping $(I-t A)$ is nonexpansive from $C$ into $H$, if $0 \leq t \leq 2 \alpha$.

\section{Main results}

Theorem 3.1 Let $C$ be a nonempty closed convex subset of a real Hilbert space $H$. Let $F: C \rightarrow H$ be a k-Lipschitzian and $\eta$-strongly monotone operator, and $g: C \rightarrow H$ be a $\rho$-contraction mapping. Let $S: C \rightarrow C$ be a nonexpansive mapping and $A: C \rightarrow H$ be an $\alpha$-inverse strongly monotone mapping. Let $\mathcal{T}=\left\{T_{n}\right\}$ be a sequence of nearly nonexpansive mappings from $C$ into itself with respect to a sequence $\left\{a_{n}\right\}$ such that $\sum_{n=1}^{\infty} \mathcal{D}_{B}\left(T_{n}, T_{n+1}\right)<\infty$ for all $B \in \mathcal{B}(C)$ and $F(\mathcal{T}) \cap \Omega(C, A) \neq \emptyset$ and let $T$ be a mapping from $C$ into itself defined by $T x=\lim _{n \rightarrow \infty} T_{n} x$ for all $x \in C$. Suppose that $F(T)=F(\mathcal{T}), 0<\mu<\frac{2 \eta}{k^{2}}$ and $0<\gamma \leq \tau$, where 
$\tau=1-\sqrt{1-\mu\left(2 \eta-\mu k^{2}\right)}$. Assume that $\Omega$, the set of solutions of the hierarchical variational inequality of finding $z^{*} \in F(\mathcal{T}) \cap \Omega(C, A)$ such that

$$
\left\langle(\mu F-\gamma S) z^{*}, z-z^{*}\right\rangle \geq 0, \quad \forall z \in F(\mathcal{T}) \cap \Omega(C, A),
$$

is nonempty. Consider the sequence $\left\{x_{n}\right\}$ in $C$ for arbitrary $x_{1} \in C$, generated by the following iterative process:

$$
\left\{\begin{array}{l}
x_{1} \in C, \\
y_{n}=T_{n} P_{C}\left[x_{n}-t_{n} A x_{n}\right], \\
x_{n+1}=P_{C}\left[\lambda_{n} \gamma\left(\alpha_{n} g\left(x_{n}\right)+\left(1-\alpha_{n}\right) S x_{n}\right)+\left(I-\lambda_{n} \mu F\right) y_{n}\right]
\end{array}\right.
$$

for all $n \in \mathbb{N}$, where $\left\{\alpha_{n}\right\},\left\{\lambda_{n}\right\}$ are sequences in $(0,1)$, and $\left\{t_{n}\right\}$ is a sequence in $[a, b]$ (for some $a, b$ with $0<a<b<2 \alpha)$ satisfying the following conditions:

(i) $\lim _{n \rightarrow \infty} \lambda_{n}=0, \lim _{n \rightarrow \infty} \alpha_{n}=0$ and $\sum_{n=1}^{\infty} \alpha_{n} \lambda_{n}=\infty$;

(ii) $\lim _{n \rightarrow \infty} \frac{\left|\alpha_{n} \lambda_{n}-\alpha_{n-1} \lambda_{n-1}\right|}{\alpha_{n} \lambda_{n}^{2}}=0$ and $\lim _{n \rightarrow \infty} \frac{\left|\lambda_{n}-\lambda_{n-1}\right|}{\alpha_{n} \lambda_{n}^{2} \lambda_{n-1}}=0$;

(iii) $\lim _{n \rightarrow \infty} \frac{\mathcal{D}_{B}\left(T_{n}, T_{n+1}\right)}{\alpha_{n+1} \lambda_{n+1}^{2}}=0$ for each $B \in \mathcal{B}(C)$ and $\sum_{n=1}^{\infty}\left|t_{n+1}-t_{n}\right|<\infty$;

(iv) $\lim _{n \rightarrow \infty} \frac{\lambda_{n}^{\frac{1}{\theta}}}{\alpha_{n}}=0, \lim _{n \rightarrow \infty} \frac{a_{n}}{\alpha_{n} \lambda_{n}^{2}}=0$ and $\lim _{n \rightarrow \infty} \frac{\left|t_{n}-t_{n-1}\right|}{\alpha_{n} \lambda_{n}^{2}}=0$;

(v) there are constants $\bar{k}>0$ and $\theta>0$ satisfying

$$
\left\|x-T_{n} x\right\| \geq \bar{k}[d(x, F(\mathcal{T}) \cap \Omega(C, A))]^{\theta}, \quad \forall x \in C \text { and } n \in \mathbb{N} .
$$

If the generated sequence $\left\{x_{n}\right\}$ is bounded and $\lim _{n \rightarrow \infty} \frac{\left\|x_{n}-P_{C}\left[x_{n}-t_{n} A x_{n}\right]\right\|}{\lambda_{n}}=0$, then it converges strongly to the point $x^{*} \in F(\mathcal{T}) \cap \Omega(C, A)$, where $x^{*}$ is the unique solution of the triple hierarchical variational inequality of finding $x^{*} \in \Omega$ such that

$$
\left\langle(\mu F-\gamma g) x^{*}, x-x^{*}\right\rangle \geq 0, \quad \forall x \in \Omega \text {. }
$$

Proof First of all, we assume that $\left\{x_{n}\right\}$ is bounded and $\lim _{n \rightarrow \infty} \frac{\left\|x_{n}-P_{C}\left[x_{n}-t_{n} A x_{n}\right]\right\|}{\lambda_{n}}=0$. We divide the proof into several steps.

Step 1. $\lim _{n \rightarrow \infty}\left\|x_{n+1}-x_{n}\right\|=0$.

Set $u_{n}=\lambda_{n} \gamma\left(\alpha_{n} g\left(x_{n}\right)+\left(1-\alpha_{n}\right) S x_{n}\right)+\left(I-\lambda_{n} \mu F\right) y_{n}$ and $\gamma_{n}=(1-\rho) \gamma \lambda_{n} \alpha_{n}$. Then, we have

$$
\begin{aligned}
u_{n}-u_{n-1}= & \alpha_{n} \lambda_{n} \gamma\left[g\left(x_{n}\right)-g\left(x_{n-1}\right)\right]+\lambda_{n}\left(1-\alpha_{n}\right) \gamma\left(S x_{n}-S x_{n-1}\right) \\
& +\left[\left(I-\lambda_{n} \mu F\right) y_{n}-\left(I-\lambda_{n} \mu F\right) y_{n-1}\right] \\
& +\left(\alpha_{n} \lambda_{n}-\alpha_{n-1} \lambda_{n-1}\right) \gamma\left[g\left(x_{n-1}\right)-S x_{n-1}\right] \\
& +\left(\lambda_{n}-\lambda_{n-1}\right)\left(\gamma S x_{n-1}-\mu F y_{n-1}\right)
\end{aligned}
$$

From (3.2), we have

$$
\begin{aligned}
\left\|x_{n+1}-x_{n}\right\| & =\left\|P_{C}\left(u_{n}\right)-P_{C}\left(u_{n-1}\right)\right\| \\
& \leq\left\|u_{n}-u_{n-1}\right\| \\
& \leq \alpha_{n} \lambda_{n} \gamma\left\|g\left(x_{n}\right)-g\left(x_{n-1}\right)\right\|+\lambda_{n}\left(1-\alpha_{n}\right) \gamma\left\|S x_{n}-S x_{n-1}\right\|
\end{aligned}
$$




$$
\begin{aligned}
& +\left\|\left(I-\lambda_{n} \mu F\right) y_{n}-\left(I-\lambda_{n} \mu F\right) y_{n-1}\right\| \\
& +\left|\alpha_{n} \lambda_{n}-\alpha_{n-1} \lambda_{n-1}\right| \gamma\left\|g\left(x_{n-1}\right)-S x_{n-1}\right\| \\
& +\left|\lambda_{n}-\lambda_{n-1}\right|\left\|\gamma S x_{n-1}-\mu F y_{n-1}\right\| \\
\leq & \alpha_{n} \lambda_{n} \gamma \rho\left\|x_{n}-x_{n-1}\right\|+\lambda_{n}\left(1-\alpha_{n}\right) \gamma\left\|x_{n}-x_{n-1}\right\| \\
& +\left(1-\lambda_{n} \tau\right)\left\|y_{n}-y_{n-1}\right\|+\left|\alpha_{n} \lambda_{n}-\alpha_{n-1} \lambda_{n-1}\right| M+\left|\lambda_{n}-\lambda_{n-1}\right| M,
\end{aligned}
$$

where $M$ is a constant such that

$$
M=\sup _{n \in \mathbb{N}}\left\{\gamma\left\|g\left(x_{n}\right)-S\left(x_{n}\right)\right\|+\left\|\gamma S x_{n}-\mu F y_{n}\right\|\right\} .
$$

Set $z_{n}:=P_{C}\left(x_{n}-t_{n} A x_{n}\right)$ and $B=\left\{z_{n}\right\}$. Since $\left\{x_{n}\right\}$ is bounded, it follows that $B \in \mathcal{B}(C)$. Now, we have

$$
\begin{aligned}
\left\|y_{n+1}-y_{n}\right\|= & \left\|T_{n+1} P_{C}\left(x_{n+1}-t_{n+1} A x_{n+1}\right)-T_{n} P_{C}\left(x_{n}-t_{n} A x_{n}\right)\right\| \\
\leq & \left\|T_{n+1} P_{C}\left(x_{n+1}-t_{n+1} A x_{n+1}\right)-T_{n+1} P_{C}\left(x_{n}-t_{n} A x_{n}\right)\right\| \\
& +\left\|T_{n+1} P_{C}\left(x_{n}-t_{n} A x_{n}\right)-T_{n} P_{C}\left(x_{n}-t_{n} A x_{n}\right)\right\| \\
\leq & \left\|P_{C}\left(x_{n+1}-t_{n+1} A x_{n+1}\right)-P_{C}\left(x_{n}-t_{n} A x_{n}\right)\right\| \\
& +\mathcal{D}_{B}\left(T_{n+1}, T_{n}\right)+a_{n+1} \\
\leq & \left\|\left(x_{n+1}-t_{n+1} A x_{n+1}\right)-\left(x_{n}-t_{n} A x_{n}\right)\right\|+\mathcal{D}_{B}\left(T_{n+1}, T_{n}\right)+a_{n+1} \\
\leq & \left\|x_{n+1}-x_{n}\right\|+\left|t_{n+1}-t_{n}\right|\left\|A x_{n}\right\|+\mathcal{D}_{B}\left(T_{n+1}, T_{n}\right)+a_{n+1} .
\end{aligned}
$$

From (3.4) and (3.5), we obtain

$$
\begin{aligned}
\left\|x_{n+1}-x_{n}\right\| \leq & \alpha_{n} \lambda_{n} \gamma \rho\left\|x_{n}-x_{n-1}\right\|+\lambda_{n}\left(1-\alpha_{n}\right) \gamma\left\|x_{n}-x_{n-1}\right\| \\
& +\left|\alpha_{n} \lambda_{n}-\alpha_{n-1} \lambda_{n-1}\right| M+\left|\lambda_{n}-\lambda_{n-1}\right| M+\left(1-\lambda_{n} \tau\right)\left[\left\|x_{n}-x_{n-1}\right\|\right. \\
& \left.+\mathcal{D}_{B}\left(T_{n}, T_{n-1}\right)+\left|t_{n}-t_{n-1}\right|\left\|A x_{n-1}\right\|+a_{n}\right] \\
= & \left(1-(1-\rho) \gamma \lambda_{n} \alpha_{n}\right)\left\|x_{n}-x_{n-1}\right\|+M\left(\left|\alpha_{n} \lambda_{n}-\alpha_{n-1} \lambda_{n-1}\right|\right. \\
& \left.+\left|\lambda_{n}-\lambda_{n-1}\right|\right)+\left(1-\lambda_{n} \tau\right)\left[\mathcal{D}_{B}\left(T_{n}, T_{n-1}\right)+\left|t_{n}-t_{n-1}\right|\left\|A x_{n-1}\right\|+a_{n}\right] \\
\leq & \left(1-\gamma_{n}\right)\left\|x_{n}-x_{n-1}\right\|+M\left(\left|\alpha_{n} \lambda_{n}-\alpha_{n-1} \lambda_{n-1}\right|+\left|\lambda_{n}-\lambda_{n-1}\right|\right) \\
& +\mathcal{D}_{B}\left(T_{n}, T_{n-1}\right)+N\left|t_{n}-t_{n-1}\right|+a_{n} \\
\leq & \left(1-\gamma_{n}\right)\left\|x_{n}-x_{n-1}\right\|+\gamma_{n}\left[M\left(\frac{\left|\alpha_{n} \lambda_{n}-\alpha_{n-1} \lambda_{n-1}\right|+\left|\lambda_{n}-\lambda_{n-1}\right|}{\gamma_{n}}\right)\right. \\
& \left.+\frac{\mathcal{D}_{B}\left(T_{n}, T_{n-1}\right)}{\gamma_{n}}+\frac{N\left|t_{n}-t_{n-1}\right|}{\gamma_{n}}+\frac{a_{n}}{\gamma_{n}}\right],
\end{aligned}
$$

where $N=\sup _{n \in \mathbb{N}}\left\{\left\|A x_{n}\right\|\right\}$. Note that $\lim _{n \rightarrow \infty} \frac{a_{n}}{\alpha_{n} \lambda_{n}}=0$ and $\sum_{n=1}^{\infty} \alpha_{n} \lambda_{n}=\infty$. Therefore, from conditions (ii), (iii), and Lemma 2.4, we have $\lim _{n \rightarrow \infty}\left\|x_{n+1}-x_{n}\right\|=0$.

Step 2. $\left\|A x_{n}-A u\right\| \rightarrow 0$ for $u \in F(\mathcal{T}) \cap \Omega(C, A)$ and $\frac{\left\|x_{n+1}-x_{n}\right\|}{\lambda_{n}} \rightarrow 0$ as $n \rightarrow \infty$. 
Set $d_{n}=2 a_{n}\left\|z_{n}-u\right\|+a_{n}^{2}$ and $\varepsilon_{n}=2 \lambda_{n}\left\|\gamma\left(\alpha_{n} g\left(x_{n}\right)+\left(1-\alpha_{n}\right) S x_{n}\right)-\mu F u\right\|\left\|y_{n}-u\right\|+d_{n}$. One can observe that

$$
\begin{aligned}
\left\|z_{n}-u\right\|^{2} & =\left\|P_{C}\left(x_{n}-t_{n} A x_{n}\right)-P_{C}\left(u-t_{n} A u\right)\right\|^{2} \\
& \leq\left\|\left(x_{n}-t_{n} A x_{n}\right)-\left(u-t_{n} A u\right)\right\|^{2} \\
& =\left\|\left(x_{n}-u\right)-t_{n}\left(A x_{n}-A u\right)\right\|^{2} \\
& \leq\left\|x_{n}-u\right\|^{2}-2 t_{n}\left\langle x_{n}-u, A x_{n}-A u\right\rangle+t_{n}^{2}\left\|A x_{n}-A u\right\|^{2} \\
& \leq\left\|x_{n}-u\right\|^{2}-t_{n}\left(2 \alpha-t_{n}\right)\left\|A x_{n}-A u\right\|^{2} \\
& \leq\left\|x_{n}-u\right\|^{2}-a(2 \alpha-b)\left\|A x_{n}-A u\right\|^{2} .
\end{aligned}
$$

We also have

$$
\begin{aligned}
\left\|y_{n}-u\right\|^{2} & =\left\|T_{n} z_{n}-T_{n} u\right\|^{2} \\
& \leq\left(\left\|z_{n}-u\right\|+a_{n}\right)^{2} \\
& \leq\left\|z_{n}-u\right\|^{2}+2 a_{n}\left\|z_{n}-u\right\|+a_{n}^{2} \\
& =\left\|z_{n}-u\right\|^{2}+d_{n} .
\end{aligned}
$$

From (3.2), we have

$$
\begin{aligned}
\left\|x_{n+1}-u\right\|^{2}= & \left\|P_{C}\left[\lambda_{n} \gamma\left(\alpha_{n} g\left(x_{n}\right)+\left(1-\alpha_{n}\right) S x_{n}\right)+\left(I-\lambda_{n} \mu F\right) y_{n}\right]-P_{C}(u)\right\|^{2} \\
\leq & \left\|\lambda_{n} \gamma\left(\alpha_{n} g\left(x_{n}\right)+\left(1-\alpha_{n}\right) S x_{n}\right)+\left(I-\lambda_{n} \mu F\right) y_{n}-u\right\|^{2} \\
= & \| \lambda_{n}\left(\gamma\left(\alpha_{n} g\left(x_{n}\right)+\left(1-\alpha_{n}\right) S x_{n}\right)-\mu F u\right)+\left(I-\lambda_{n} \mu F\right)\left(y_{n}\right) \\
& -\left(I-\lambda_{n} \mu F\right)(u) \|^{2} \\
\leq & {\left[\lambda_{n}\left\|\gamma\left(\alpha_{n} g\left(x_{n}\right)+\left(1-\alpha_{n}\right) S x_{n}\right)-\mu F u\right\|+\left(1-\lambda_{n} \tau\right)\left\|y_{n}-u\right\|\right]^{2} } \\
\leq & \lambda_{n}\left\|\gamma\left(\alpha_{n} g\left(x_{n}\right)+\left(1-\alpha_{n}\right) S x_{n}\right)-\mu F u\right\|^{2} \\
& +\left(1-\lambda_{n} \tau\right)\left(\left\|z_{n}-u\right\|^{2}+d_{n}\right) \\
& +2 \lambda_{n}\left(1-\lambda_{n} \tau\right)\left\|\gamma\left(\alpha_{n} g\left(x_{n}\right)+\left(1-\alpha_{n}\right) S x_{n}\right)-\mu F u\right\|\left\|y_{n}-u\right\| \\
\leq & \lambda_{n}\left\|\gamma\left(\alpha_{n} g\left(x_{n}\right)+\left(1-\alpha_{n}\right) S x_{n}\right)-\mu F u\right\|^{2}+\left\|z_{n}-u\right\|^{2}+\varepsilon_{n} \\
\leq & \lambda_{n}\left\|\gamma\left(\alpha_{n} g\left(x_{n}\right)+\left(1-\alpha_{n}\right) S x_{n}\right)-\mu F u\right\|^{2}+\left\|x_{n}-u\right\|^{2} \\
& -a(2 \alpha-b)\left\|A x_{n}-A u\right\|^{2}+\varepsilon_{n} .
\end{aligned}
$$

Thus, we get

$$
\begin{aligned}
a(2 \alpha-b)\left\|A x_{n}-A u\right\|^{2} \leq & \lambda_{n}\left\|\gamma\left(\alpha_{n} g\left(x_{n}\right)+\left(1-\alpha_{n}\right) S x_{n}\right)-\mu F u\right\|^{2} \\
& +\left(\left\|x_{n}-u\right\|^{2}-\left\|x_{n+1}-u\right\|^{2}\right)+\varepsilon_{n} \\
\leq & \lambda_{n}\left\|\gamma\left(\alpha_{n} g\left(x_{n}\right)+\left(1-\alpha_{n}\right) S x_{n}\right)-\mu F u\right\|^{2} \\
& +\left\|x_{n}-x_{n+1}\right\|\left(\left\|x_{n}-u\right\|+\left\|x_{n+1}-u\right\|\right)+\varepsilon_{n} .
\end{aligned}
$$


Since $\lambda_{n} \rightarrow 0, \varepsilon_{n} \rightarrow 0$ and $\left\|x_{n+1}-x_{n}\right\| \rightarrow 0$ as $n \rightarrow \infty$, we obtain $\left\|A x_{n}-A u\right\| \rightarrow 0$ as $n \rightarrow \infty$. From (3.6), we have

$$
\begin{aligned}
\frac{\left\|x_{n+1}-x_{n}\right\|}{\lambda_{n}} \leq & \left(1-\gamma_{n}\right) \frac{\left\|x_{n}-x_{n-1}\right\|}{\lambda_{n}}+\frac{M\left(\left|\alpha_{n} \lambda_{n}-\alpha_{n-1} \lambda_{n-1}\right|+\left|\lambda_{n}-\lambda_{n-1}\right|\right)}{\lambda_{n}} \\
& +\frac{\mathcal{D}_{B}\left(T_{n}, T_{n-1}\right)}{\lambda_{n}}+\frac{N\left|t_{n}-t_{n-1}\right|}{\lambda_{n}}+\frac{a_{n}}{\lambda_{n}} \\
= & \left(1-\gamma_{n}\right) \frac{\left\|x_{n}-x_{n-1}\right\|}{\lambda_{n-1}}+\left(1-\gamma_{n}\right)\left(\frac{\left\|x_{n}-x_{n-1}\right\|}{\lambda_{n}}-\frac{\left\|x_{n}-x_{n-1}\right\|}{\lambda_{n-1}}\right) \\
& +\frac{M\left(\left|\alpha_{n} \lambda_{n}-\alpha_{n-1} \lambda_{n-1}\right|+\left|\lambda_{n}-\lambda_{n-1}\right|\right)}{\lambda_{n}} \\
& +\frac{\mathcal{D}_{B}\left(T_{n}, T_{n-1}\right)}{\lambda_{n}}+\frac{N\left|t_{n}-t_{n-1}\right|}{\lambda_{n}}+\frac{a_{n}}{\lambda_{n}} \\
\leq & \left(1-\gamma_{n}\right) \frac{\left\|x_{n}-x_{n-1}\right\|}{\lambda_{n-1}}+\alpha_{n} \lambda_{n}\left\|x_{n}-x_{n-1}\right\| \frac{1}{\alpha_{n} \lambda_{n}}\left|\frac{1}{\lambda_{n}}-\frac{1}{\lambda_{n-1}}\right| \\
& +\frac{M \alpha_{n} \lambda_{n}\left(\left|\alpha_{n} \lambda_{n}-\alpha_{n-1} \lambda_{n-1}\right|+\left|\lambda_{n}-\lambda_{n-1}\right|\right)}{\alpha_{n} \lambda_{n}^{2}} \\
& +\alpha_{n} \lambda_{n}\left(\frac{\mathcal{D}_{B}\left(T_{n}, T_{n-1}\right)}{\alpha_{n} \lambda_{n}^{2}}+\frac{N\left|t_{n}-t_{n-1}\right|}{\alpha_{n} \lambda_{n}^{2}}+\frac{a_{n}}{\alpha_{n} \lambda_{n}^{2}}\right) .
\end{aligned}
$$

Noticing that $\lim _{n \rightarrow \infty} \frac{a_{n}}{\alpha_{n} \lambda_{n}^{2}}=0, \lim _{n \rightarrow \infty} \frac{\left|t_{n}-t_{n-1}\right|}{\alpha_{n} \lambda_{n}^{2}}=0$, and $\sum_{n=1}^{\infty} \alpha_{n} \lambda_{n}=\infty$. Thus, using conditions (ii) and (iii), and applying Lemma 2.4 , we have

$$
\lim _{n \rightarrow \infty} \frac{\left\|x_{n+1}-x_{n}\right\|}{\lambda_{n}}=0
$$

Step 3. $\left\|x_{n}-z_{n}\right\| \rightarrow 0$ as $n \rightarrow \infty$.

Let $u \in F(\mathcal{T}) \cap \Omega(C, A)$. Then using Lemma 2.1(iv), we have

$$
\begin{aligned}
\left\|z_{n}-u\right\|^{2}= & \left\|P_{C}\left(x_{n}-t_{n} A x_{n}\right)-P_{C}\left(u-t_{n} A u\right)\right\|^{2} \\
\leq & \left\langle\left(x_{n}-t_{n} A x_{n}\right)-\left(u-t_{n} A u\right), z_{n}-u\right\rangle \\
= & \frac{1}{2}\left[\left\|\left(x_{n}-t_{n} A x_{n}\right)-\left(u-t_{n} A u\right)\right\|^{2}+\left\|z_{n}-u\right\|^{2}\right. \\
& \left.-\left\|\left(x_{n}-t_{n} A x_{n}\right)-\left(u-t_{n} A u\right)-\left(z_{n}-u\right)\right\|^{2}\right] \\
\leq & \frac{1}{2}\left[\left\|x_{n}-u\right\|^{2}+\left\|z_{n}-u\right\|^{2}-\left\|\left(x_{n}-z_{n}\right)-t_{n}\left(A x_{n}-A u\right)\right\|^{2}\right] .
\end{aligned}
$$

It follows that

$$
\begin{aligned}
\left\|z_{n}-u\right\|^{2} \leq & \left\|x_{n}-u\right\|^{2}-\left\|x_{n}-z_{n}\right\|^{2} \\
& +2 t_{n}\left\langle x_{n}-z_{n}, A x_{n}-A u\right\rangle-t_{n}^{2}\left\|A x_{n}-A u\right\|^{2} .
\end{aligned}
$$

From (3.7) and (3.9), we have

$$
\begin{aligned}
\left\|x_{n+1}-u\right\|^{2} \leq & \lambda_{n}\left\|\gamma\left(\alpha_{n} g\left(x_{n}\right)+\left(1-\alpha_{n}\right) S x_{n}\right)-\mu F u\right\|^{2}+\left\|x_{n}-u\right\|^{2} \\
& -\left\|x_{n}-z_{n}\right\|^{2}+2 t_{n}\left\langle x_{n}-z_{n}, A x_{n}-A u\right\rangle-t_{n}^{2}\left\|A x_{n}-A u\right\|^{2}+\varepsilon_{n},
\end{aligned}
$$


which gives

$$
\begin{aligned}
\left\|x_{n}-z_{n}\right\|^{2} \leq & \lambda_{n}\left\|\gamma\left(\alpha_{n} g\left(x_{n}\right)+\left(1-\alpha_{n}\right) S x_{n}\right)-\mu F u\right\|^{2} \\
& +\left(\left\|x_{n}-u\right\|^{2}-\left\|x_{n+1}-u\right\|^{2}\right) \\
& +2 t_{n}\left\langle x_{n}-z_{n}, A x_{n}-A u\right\rangle-t_{n}^{2}\left\|A x_{n}-A u\right\|^{2}+\varepsilon_{n} \\
\leq & \lambda_{n}\left\|\gamma\left(\alpha_{n} g\left(x_{n}\right)+\left(1-\alpha_{n}\right) S x_{n}\right)-\mu F u\right\|^{2} \\
& +\left(\left\|x_{n}-u\right\|+\left\|x_{n+1}-u\right\|\right)\left\|x_{n}-x_{n+1}\right\| \\
& +2 t_{n}\left\|x_{n}-z_{n}\right\|\left\|A x_{n}-A u\right\|-t_{n}^{2}\left\|A x_{n}-A u\right\|^{2}+\varepsilon_{n} .
\end{aligned}
$$

We have $\left\|x_{n+1}-x_{n}\right\| \rightarrow 0, \lambda_{n} \rightarrow 0, \varepsilon_{n} \rightarrow 0$, and $\left\|A x_{n}-A u\right\| \rightarrow 0$ as $n \rightarrow \infty$. Therefore, we have $\left\|x_{n}-z_{n}\right\| \rightarrow 0$ as $n \rightarrow \infty$.

Step 4. $\left\|x_{n}-T x_{n}\right\| \rightarrow 0$ as $n \rightarrow \infty$.

Since $y_{n}=T_{n} z_{n}$, we get

$$
\begin{aligned}
\left\|x_{n+1}-T_{n} z_{n}\right\| & =\left\|P_{C}\left[\lambda_{n} \gamma\left(\alpha_{n} g\left(x_{n}\right)+\left(1-\alpha_{n}\right) S x_{n}\right)+\left(I-\lambda_{n} \mu F\right) y_{n}\right]-P_{C}\left(T_{n} z_{n}\right)\right\| \\
& \leq\left\|\lambda_{n} \gamma\left(\alpha_{n} g\left(x_{n}\right)+\left(1-\alpha_{n}\right) S x_{n}\right)+\left(I-\lambda_{n} \mu F\right) y_{n}-T_{n} z_{n}\right\| \\
& =\left\|\lambda_{n} \gamma\left(\alpha_{n} g\left(x_{n}\right)+\left(1-\alpha_{n}\right) S x_{n}\right)+y_{n}-\lambda_{n} \mu F y_{n}-T_{n} z_{n}\right\| \\
& =\lambda_{n}\left\|\gamma\left(\alpha_{n} g\left(x_{n}\right)+\left(1-\alpha_{n}\right) S x_{n}\right)-\mu F y_{n}\right\| \rightarrow 0 \quad \text { as } n \rightarrow \infty .
\end{aligned}
$$

It follows that

$$
\begin{aligned}
\left\|x_{n}-y_{n}\right\| & =\left\|x_{n}-T_{n} z_{n}\right\| \\
& \leq\left\|x_{n}-x_{n+1}\right\|+\left\|x_{n+1}-T_{n} z_{n}\right\| \rightarrow 0 \quad \text { as } n \rightarrow \infty .
\end{aligned}
$$

Also, we get

$$
\left\|z_{n}-T_{n} z_{n}\right\| \leq\left\|z_{n}-x_{n}\right\|+\left\|x_{n}-T_{n} z_{n}\right\| \rightarrow 0 \quad \text { as } n \rightarrow \infty .
$$

Note that

$$
\begin{aligned}
\left\|x_{n}-T_{n} x_{n}\right\| & \leq\left\|x_{n}-T_{n} z_{n}\right\|+\left\|T_{n} z_{n}-T_{n} x_{n}\right\| \\
& \leq\left\|x_{n}-T_{n} z_{n}\right\|+\left\|z_{n}-x_{n}\right\|+a_{n} \rightarrow 0 \quad \text { as } n \rightarrow \infty .
\end{aligned}
$$

Thus,

$$
\begin{aligned}
\left\|T x_{n}-x_{n}\right\| & \leq\left\|T x_{n}-T z_{n}\right\|+\left\|T z_{n}-T_{n} z_{n}\right\|+\left\|T_{n} z_{n}-x_{n}\right\| \\
& \leq\left\|x_{n}-z_{n}\right\|+\mathcal{D}_{B}\left(T_{n}, T\right)+\left\|T_{n} z_{n}-x_{n}\right\| \rightarrow 0 \quad \text { as } n \rightarrow \infty .
\end{aligned}
$$

Step 5. $\omega_{w}\left(\left\{x_{n}\right\}\right) \subset F(\mathcal{T}) \cap \Omega(C, A)$.

Note that $A$ is an $\alpha$-inverse strongly monotone mapping so that it is $\frac{1}{\alpha}$-Lipschitz continuous. Therefore, we have

$$
\lim _{n \rightarrow \infty}\left\|A z_{n}-A x_{n}\right\|=0
$$


Since $\left\{x_{n}\right\}$ is a bounded sequence in $C$, there exists a subsequence $\left\{x_{n_{k}}\right\}$ of $\left\{x_{n}\right\}$ which converges weakly to some $\hat{x}$ in $C$. Since $\lim _{n \rightarrow \infty}\left\|x_{n}-T x_{n}\right\|=0$, it follows, from the demiclosedness principle of nonexpansive mappings, that $\hat{x} \in F(\mathcal{T})$. Now, let us show that $\hat{x} \in \Omega(C, A)$.

Let

$$
\mathbb{A} v= \begin{cases}A v+N_{C}(v), & \text { if } v \in C, \\ \emptyset, & \text { if } v \notin C .\end{cases}
$$

Note that $\mathbb{A}$ is maximal monotone and $0 \in \mathbb{A} v$ if and only if $v \in \Omega(C, A)$. Let $(v, w) \in G(\mathbb{A})$, the graph of $\mathbb{A}$. Then, we have $w \in \mathbb{A} v=A v+N_{C}(v)$ and hence $w-A v \in N_{C}(v)$. Thus, we have

$$
\langle w-A v, v-u\rangle \geq 0 \quad \text { for all } u \in C .
$$

On the other hand, from $z_{n}=P_{C}\left(x_{n}-t_{n} A x_{n}\right)$ and $v \in C$, we have

$$
\left\langle x_{n}-t_{n} A x_{n}-z_{n}, z_{n}-v\right\rangle \geq 0,
$$

and hence

$$
\left\langle v-z_{n}, \frac{z_{n}-x_{n}}{t_{n}}+A x_{n}\right\rangle \geq 0
$$

Therefore, from $w-A v \in N_{C}(v)$ and $z_{n_{i}} \in C$, we have

$$
\begin{aligned}
\left\langle v-z_{n_{i}}, w\right\rangle \geq & \left\langle v-z_{n_{i}}, A v\right\rangle \\
\geq & \left\langle v-z_{n_{i}}, A v\right\rangle-\left\langle v-z_{n_{i}}, \frac{z_{n_{i}}-x_{n_{i}}}{t_{n_{i}}}+A x_{n_{i}}\right\rangle \\
= & \left\langle v-z_{n_{i}}, A v-A z_{n_{i}}\right\rangle+\left\langle v-z_{n_{i}}, A z_{n_{i}}-A x_{n_{i}}\right\rangle \\
& -\left\langle v-z_{n_{i}}, \frac{z_{n_{i}}-x_{n_{i}}}{t_{n_{i}}}\right\rangle \\
\geq & \left\langle v-z_{n_{i}}, A z_{n_{i}}-A x_{n_{i}}\right\rangle-\left\langle v-z_{n_{i}}, \frac{z_{n_{i}}-x_{n_{i}}}{t_{n_{i}}}\right\rangle .
\end{aligned}
$$

Letting limit $n_{i} \rightarrow \infty$ we obtain $\langle v-\hat{x}, w\rangle \geq 0$. Thus, $\hat{x} \in \mathbb{A}^{-1} 0$ together with the maximal monotonicity of $\mathbb{A}$ imply $\hat{x} \in \Omega(C, A)$.

Step 6. $\lim \sup _{n \rightarrow \infty}\left\langle(\mu F-\gamma g) x^{*}, x_{n}-x^{*}\right\rangle \geq 0$.

From (3.2), we have

$$
x_{n+1}=P_{C}\left(u_{n}\right)-u_{n}+\lambda_{n} \gamma\left(\alpha_{n} g\left(x_{n}\right)+\left(1-\alpha_{n}\right) S x_{n}\right)+\left(I-\lambda_{n} \mu F\right) y_{n} .
$$

Therefore, we have

$$
\begin{aligned}
x_{n}-x_{n+1}= & u_{n}-P_{C}\left(u_{n}\right)+\alpha_{n} \lambda_{n}(\mu F-\gamma g) x_{n}+\lambda_{n}\left(1-\alpha_{n}\right)(\mu F-\gamma S) x_{n} \\
& +\left(1-\lambda_{n}\right)\left(x_{n}-y_{n}\right)+\lambda_{n}\left[(I-\mu F) x_{n}-(I-\mu F) y_{n}\right] .
\end{aligned}
$$


Set $v_{n}:=\frac{x_{n}-x_{n+1}}{\lambda_{n}\left(1-\alpha_{n}\right)}, \forall n \in \mathbb{N}$. Note $x_{n}=P_{C}\left(u_{n-1}\right)$. Then, we have

$$
\begin{aligned}
v_{n}= & \frac{u_{n}-P_{C}\left(u_{n}\right)}{\lambda_{n}\left(1-\alpha_{n}\right)}+\frac{\alpha_{n}}{1-\alpha_{n}}(\mu F-\gamma g) x_{n}+(\mu F-\gamma S) x_{n} \\
& +\frac{\left(1-\lambda_{n}\right)}{\lambda_{n}\left(1-\alpha_{n}\right)}\left(x_{n}-y_{n}\right)+\frac{1}{1-\alpha_{n}}\left[(I-\mu F) x_{n}-(I-\mu F) y_{n}\right] .
\end{aligned}
$$

Let $w \in F(\mathcal{T}) \cap \Omega(C, A)$. Observe that

$$
\begin{aligned}
\left\langle v_{n}, x_{n}-w\right\rangle= & \frac{1}{\lambda_{n}\left(1-\alpha_{n}\right)}\left\langle u_{n}-P_{C}\left(u_{n}\right), P_{C}\left(u_{n-1}\right)-w\right\rangle \\
& +\frac{\alpha_{n}}{1-\alpha_{n}}\left\langle(\mu F-\gamma g) x_{n}, x_{n}-w\right\rangle \\
& +\left\langle(\mu F-\gamma S) x_{n}, x_{n}-w\right\rangle+\frac{1-\lambda_{n}}{\lambda_{n}\left(1-\alpha_{n}\right)}\left\langle x_{n}-y_{n}, x_{n}-w\right\rangle \\
& +\frac{1}{1-\alpha_{n}}\left\langle(I-\mu F) x_{n}-(I-\mu F) y_{n}, x_{n}-w\right\rangle \\
= & \frac{1}{\lambda_{n}\left(1-\alpha_{n}\right)}\left\langle u_{n}-P_{C}\left(u_{n}\right), P_{C}\left(u_{n}\right)-w\right\rangle \\
& +\frac{1}{\lambda_{n}\left(1-\alpha_{n}\right)}\left\langle u_{n}-P_{C}\left(u_{n}\right), P_{C}\left(u_{n-1}\right)-P_{C}\left(u_{n}\right)\right\rangle \\
& +\left\langle(\mu F-\gamma S) w, x_{n}-w\right\rangle+\left\langle(\mu F-\gamma S) x_{n}-(\mu F-\gamma S) w, x_{n}-w\right\rangle \\
& +\frac{1-\lambda_{n}}{\lambda_{n}\left(1-\alpha_{n}\right)}\left\langle x_{n}-y_{n}, x_{n}-w\right\rangle+\frac{\alpha_{n}}{1-\alpha_{n}}\left\langle(\mu F-\gamma g) x_{n}, x_{n}-w\right\rangle \\
& +\frac{1}{1-\alpha_{n}}\left\langle(I-\mu F) x_{n}-(I-\mu F) y_{n}, x_{n}-w\right\rangle .
\end{aligned}
$$

The first and fourth terms in (3.11) are nonnegative due to the property of the projection operator given in Lemma 2.1(ii), and the monotonicity of $(\mu F-\gamma S)$, respectively. Note $x_{n+1}=P_{C}\left(u_{n}\right)$. Thus, from (3.11), we have

$$
\begin{aligned}
\left\langle v_{n}, x_{n}-w\right\rangle \geq & \frac{1}{\lambda_{n}\left(1-\alpha_{n}\right)}\left\langle u_{n}-P_{C}\left(u_{n}\right), P_{C}\left(u_{n-1}\right)-P_{C}\left(u_{n}\right)\right\rangle \\
& +\left\langle(\mu F-\gamma S) w, x_{n}-w\right\rangle+\frac{\alpha_{n}}{1-\alpha_{n}}\left\langle(\mu F-\gamma g) x_{n}, x_{n}-w\right\rangle \\
& +\frac{1}{1-\alpha_{n}}\left\langle(I-\mu F) x_{n}-(I-\mu F) y_{n}, x_{n}-w\right\rangle \\
& +\frac{1-\lambda_{n}}{\lambda_{n}\left(1-\alpha_{n}\right)}\left\langle x_{n}-y_{n}, x_{n}-w\right\rangle \\
= & \left\langle u_{n}-P_{C}\left(u_{n}\right), v_{n}\right\rangle+\left\langle(\mu F-\gamma S) w, x_{n}-w\right\rangle \\
& +\frac{\alpha_{n}}{1-\alpha_{n}}\left\langle(\mu F-\gamma g) x_{n}, x_{n}-w\right\rangle \\
& +\frac{1}{1-\alpha_{n}}\left\langle(I-\mu F) x_{n}-(I-\mu F) y_{n}, x_{n}-w\right\rangle \\
& +\frac{1-\lambda_{n}}{\lambda_{n}\left(1-\alpha_{n}\right)}\left\langle x_{n}-y_{n}, x_{n}-w\right\rangle .
\end{aligned}
$$


Noticing, from (3.10), that $\left\|x_{n}-y_{n}\right\| \rightarrow 0$, we have $\left\|(I-\mu F) x_{n}-(I-\mu F) y_{n}\right\| \rightarrow 0$. It is clear from (3.8) that $v_{n} \rightarrow 0$. By assumption $\alpha_{n} \rightarrow 0$ and the sequence $\left\{x_{n}\right\}$ is bounded; we see that $\left\{u_{n}\right\}$ is bounded. Thus, from (3.12), we have

$$
\limsup _{n \rightarrow \infty}\left\{(\mu F-\gamma S) w, x_{n}-w\right\rangle \leq 0, \quad \forall w \in F(\mathcal{T}) \cap \Omega(C, A)
$$

This is sufficient to guarantee that $\omega_{w}\left(\left\{x_{n}\right\}\right) \subseteq \Omega$, i.e., every weak limit point of the sequence $\left\{x_{n}\right\}$ solves the hierarchical variational inequality (3.1). In fact, if $\left\{x_{n_{k}}\right\}$ is a subsequence of $\left\{x_{n}\right\}$ such that $x_{n_{k}} \rightarrow \tilde{x} \in \omega_{w}\left(\left\{x_{n}\right\}\right)$, then, from (3.13), we have

$$
\langle(\mu F-\gamma S) w, \tilde{x}-w\rangle=\limsup _{n \rightarrow \infty}\left\langle(\mu F-\gamma S) w, x_{n}-w\right\rangle \leq 0, \quad \forall w \in F(\mathcal{T}) \cap \Omega(C, A),
$$

that is,

$$
\langle(\mu F-\gamma S) w, w-\tilde{x}\rangle \geq 0, \quad \forall w \in F(\mathcal{T}) \cap \Omega(C, A) .
$$

Note that $\omega_{w}\left(\left\{x_{n}\right\}\right) \subseteq F(\mathcal{T}) \cap \Omega(C, A)$. Moreover, $(\mu F-\gamma S)$ is monotone and Lipschitz continuous, and $F(\mathcal{T}) \cap \Omega(C, A) \neq \emptyset$ is closed and convex. Therefore, the inequality (3.14) is equivalent to the inequality (3.1) by the Minty lemma (see [22]). Thus, we have $\tilde{x} \in \Omega$.

Now, we choose a subsequence $\left\{x_{n_{k}}\right\}$ of $\left\{x_{n}\right\}$ satisfying

$$
\limsup _{n \rightarrow \infty}\left\langle(\mu F-\gamma g) x^{*}, x_{n}-x^{*}\right\rangle=\lim _{k \rightarrow \infty}\left\langle(\mu F-\gamma g) x^{*}, x_{n_{k}}-x^{*}\right\rangle .
$$

Without loss of generality, we may further assume that $x_{n_{k}} \rightarrow \tilde{x}$. Note that $\tilde{x} \in \Omega$. As $x^{*}$ is a solution of the triple hierarchical variational inequality (3.3), we obtain

$$
\limsup _{n \rightarrow \infty}\left\langle(\mu F-\gamma g) x^{*}, x_{n}-x^{*}\right\rangle=\left\langle(\mu F-\gamma g) x^{*}, \tilde{x}-x^{*}\right\rangle \geq 0 .
$$

Step 7. $x_{n} \rightarrow x^{*}$ as $n \rightarrow \infty$.

Noticing that $y_{n}=T_{n} z_{n}, \gamma_{n}=(1-\rho) \gamma \lambda_{n} \alpha_{n}$, and $x_{n+1}=P_{C}\left(u_{n}\right)$. Set $\chi_{n}=\alpha_{n} \lambda_{n} \chi_{n}^{\prime}+\chi_{n}^{\prime \prime}$, where $\chi_{n}^{\prime}:=\left\langle(\gamma g-\mu F) x^{*}, x_{n+1}-x^{*}\right\rangle$ and $\chi_{n}^{\prime \prime}=\lambda_{n}\left(1-\alpha_{n}\right)\left\langle(\gamma S-\mu F) x^{*}, x_{n+1}-x^{*}\right\rangle$. From (3.2), we have

$$
\begin{aligned}
\left\|x_{n+1}-x^{*}\right\|^{2}= & \left\langle u_{n}-x^{*}, x_{n+1}-x^{*}\right\rangle+\left\langle P_{C}\left(u_{n}\right)-u_{n}, P_{C}\left(u_{n}\right)-x^{*}\right\rangle \\
\leq & \left\langle u_{n}-x^{*}, x_{n+1}-x^{*}\right\rangle \\
= & \left\langle\lambda_{n} \gamma\left(\alpha_{n} g\left(x_{n}\right)+\left(1-\alpha_{n}\right) S x_{n}\right)+\left(I-\lambda_{n} \mu F\right) y_{n}-x^{*}, x_{n+1}-x^{*}\right\rangle \\
= & \left\langle\left(I-\lambda_{n} \mu F\right) y_{n}-\left(I-\lambda_{n} \mu F\right) x^{*}, x_{n+1}-x^{*}\right\rangle \\
& +\alpha_{n} \lambda_{n} \gamma\left\langle g\left(x_{n}\right)-g\left(x^{*}\right), x_{n+1}-x^{*}\right\rangle+\lambda_{n}\left(1-\alpha_{n}\right) \gamma\left\langle S x_{n}-S x^{*}, x_{n+1}-x^{*}\right\rangle \\
& +\alpha_{n} \lambda_{n}\left\langle(\gamma g-\mu F) x^{*}, x_{n+1}-x^{*}\right\rangle+\lambda_{n}\left(1-\alpha_{n}\right)\left\langle(\gamma S-\mu F) x^{*}, x_{n+1}-x^{*}\right\rangle \\
\leq & \left(1-\lambda_{n} \tau\right)\left(\left\|z_{n}-x^{*}\right\|+a_{n}\right)\left\|x_{n+1}-x^{*}\right\|+\alpha_{n} \lambda_{n} \gamma \rho\left\|x_{n}-x^{*}\right\|\left\|x_{n+1}-x^{*}\right\| \\
& +\lambda_{n}\left(1-\alpha_{n}\right) \gamma\left\|x_{n}-x^{*}\right\|\left\|x_{n+1}-x^{*}\right\|+\chi_{n} \\
\leq & \left(1-\lambda_{n} \tau\right)\left\|x_{n}-x^{*}\right\|\left\|x_{n+1}-x^{*}\right\|+\alpha_{n} \lambda_{n} \gamma \rho\left\|x_{n}-x^{*}\right\|\left\|x_{n+1}-x^{*}\right\|
\end{aligned}
$$




$$
\begin{aligned}
& +\lambda_{n}\left(1-\alpha_{n}\right) \gamma\left\|x_{n}-x^{*}\right\|\left\|x_{n+1}-x^{*}\right\|+\left(1-\lambda_{n} \tau\right) a_{n}\left\|x_{n+1}-x^{*}\right\|+\chi_{n} \\
\leq & {\left[1-\lambda_{n} \tau+\alpha_{n} \lambda_{n} \gamma \rho+\lambda_{n}\left(1-\alpha_{n}\right) \gamma\right]\left\|x_{n}-x^{*}\right\|\left\|x_{n+1}-x^{*}\right\| } \\
& +a_{n}\left\|x_{n+1}-x^{*}\right\|+\chi_{n} \\
\leq & {\left[1-\alpha_{n} \lambda_{n} \gamma(1-\rho)\right]\left\|x_{n}-x^{*}\right\|\left\|x_{n+1}-x^{*}\right\|+a_{n}\left\|x_{n+1}-x^{*}\right\|+\chi_{n} } \\
\leq & {\left[1-\alpha_{n} \lambda_{n} \gamma(1-\rho)\right] \frac{1}{2}\left(\left\|x_{n}-x^{*}\right\|^{2}+\left\|x_{n+1}-x^{*}\right\|^{2}\right) } \\
& +a_{n}\left\|x_{n+1}-x^{*}\right\|+\chi_{n} .
\end{aligned}
$$

It follows that

$$
\begin{aligned}
\left\|x_{n+1}-x^{*}\right\|^{2} & \leq \frac{1-\alpha_{n} \lambda_{n} \gamma(1-\rho)}{1+\alpha_{n} \lambda_{n} \gamma(1-\rho)}\left\|x_{n}-x^{*}\right\|^{2}+\frac{2}{1+\gamma_{n}} \chi_{n}+\frac{2 a_{n}}{1+\gamma_{n}} R \\
& \leq\left[1-\alpha_{n} \lambda_{n} \gamma(1-\rho)\right]\left\|x_{n}-x^{*}\right\|^{2}+\frac{2 \chi_{n}}{1+\gamma_{n}}+\frac{2 a_{n} R}{1+\gamma_{n}}
\end{aligned}
$$

for some $R>0$. Since $x^{*} \in \Omega$, by using condition (v) we have

$$
\begin{aligned}
\left\langle(\gamma S-\mu F) x^{*}, x_{n+1}-x^{*}\right\rangle= & \left\langle(\gamma S-\mu F) x^{*}, x_{n+1}-P_{F(\mathcal{T}) \cap \Omega(C, A)}\left(x_{n+1}\right)\right\rangle \\
& +\left\langle(\gamma S-\mu F) x^{*}, P_{F(\mathcal{T}) \cap \Omega(C, A)}\left(x_{n+1}\right)-x^{*}\right\rangle \\
\leq & \left\langle(\gamma S-\mu F) x^{*}, x_{n+1}-P_{F(\mathcal{T}) \cap \Omega(C, A)}\left(x_{n+1}\right)\right\rangle \\
\leq & \left\|(\gamma S-\mu F) x^{*}\right\| d\left(x_{n+1}, F(\mathcal{T}) \cap \Omega(C, A)\right) \\
\leq & \left\|(\gamma S-\mu F) x^{*}\right\|\left(\frac{1}{\bar{k}}\left\|x_{n+1}-T_{n} x_{n+1}\right\|\right)^{\frac{1}{\theta}} .
\end{aligned}
$$

Note that

$$
\begin{aligned}
\left\|x_{n+1}-T_{n} x_{n}\right\| & =\left\|P_{C}\left(u_{n}\right)-P_{C}\left(T_{n} x_{n}\right)\right\| \\
& \leq\left\|u_{n}-T_{n} x_{n}\right\| \\
& =\left\|\lambda_{n} \gamma\left(\alpha_{n} g\left(x_{n}\right)+\left(1-\alpha_{n}\right) S x_{n}\right)+\left(I-\lambda_{n} \mu F\right) y_{n}-T_{n} x_{n}\right\| \\
& \leq \lambda_{n}\left\|\gamma\left(\alpha_{n} g\left(x_{n}\right)+\left(1-\alpha_{n}\right) S x_{n}\right)-\mu F y_{n}\right\|+\left\|T_{n} z_{n}-T_{n} x_{n}\right\| \\
& \leq \lambda_{n}\left\|\gamma\left(\alpha_{n} g\left(x_{n}\right)+\left(1-\alpha_{n}\right) S x_{n}\right)-\mu F y_{n}\right\|+\left\|z_{n}-x_{n}\right\|+a_{n} .
\end{aligned}
$$

We observe that

$$
\begin{aligned}
\left\|x_{n+1}-T_{n} x_{n+1}\right\| \leq & \left\|x_{n+1}-T_{n} x_{n}\right\|+\left\|T_{n} x_{n}-T_{n} x_{n+1}\right\| \\
\leq & \left\|x_{n}-x_{n+1}\right\|+\left\|x_{n+1}-T_{n} x_{n}\right\|+a_{n} \\
\leq & \left\|x_{n}-x_{n+1}\right\|+\lambda_{n}\left\|\gamma\left(\alpha_{n} g\left(x_{n}\right)+\left(1-\alpha_{n}\right) S x_{n}\right)-\mu F y_{n}\right\| \\
& \quad+\left\|z_{n}-x_{n}\right\|+2 a_{n} . \\
\leq & \left\|x_{n}-x_{n+1}\right\|+\lambda_{n} M+\left\|z_{n}-x_{n}\right\|+2 a_{n} .
\end{aligned}
$$


Hence from (3.16), we get

$$
\begin{aligned}
& \left\langle(\gamma S-\mu F) x^{*}, x_{n+1}-x^{*}\right\rangle \\
& \leq\left(\frac{1}{\bar{k}}\right)^{\frac{1}{\theta}}\left\|(\gamma S-\mu F) x^{*}\right\|\left(\left\|x_{n}-x_{n+1}\right\|+M \lambda_{n}+\left\|z_{n}-x_{n}\right\|+2 a_{n}\right)^{\frac{1}{\theta}} \\
& \leq \lambda_{n}^{\frac{1}{\theta}} M^{\prime \prime}\left(1+\frac{\left\|x_{n}-x_{n+1}\right\|}{\lambda_{n}}+\frac{\left\|z_{n}-x_{n}\right\|}{\lambda_{n}}+\frac{a_{n}}{\lambda_{n}}\right)^{\frac{1}{\theta}}
\end{aligned}
$$

for some constant $M^{\prime \prime}$. Therefore from (3.15) and (3.17), we have

$$
\begin{aligned}
\left\|x_{n+1}-x^{*}\right\|^{2} \leq & {\left[1-\gamma(1-\rho) \alpha_{n} \lambda_{n}\right]\left\|x_{n}-x^{*}\right\|^{2} } \\
& +\frac{2 \alpha_{n} \lambda_{n}}{1+\gamma_{n}}\left[\chi_{n}^{\prime}+M^{\prime \prime} \frac{\lambda_{n}^{\frac{1}{\theta}}}{\alpha_{n}}\left(1+\frac{\left\|x_{n}-x_{n+1}\right\|}{\lambda_{n}}+\frac{\left\|z_{n}-x_{n}\right\|}{\lambda_{n}}+\frac{a_{n}}{\lambda_{n}}\right)^{\frac{1}{\theta}}\right]+\frac{2 a_{n} R}{1+\gamma_{n}} \\
= & \left(1-\gamma_{n}\right)\left\|x_{n}-x^{*}\right\|^{2}+\sigma_{n}+\frac{2 a_{n} R}{1+\gamma_{n}},
\end{aligned}
$$

where

$$
\sigma_{n}=\frac{2 \alpha_{n} \lambda_{n}}{1+\gamma_{n}}\left[\chi_{n}^{\prime}+M^{\prime \prime} \frac{\lambda_{n}^{\frac{1}{\theta}}}{\alpha_{n}}\left(1+\frac{\left\|x_{n}-x_{n+1}\right\|}{\lambda_{n}}+\frac{\left\|z_{n}-x_{n}\right\|}{\lambda_{n}}+\frac{a_{n}}{\lambda_{n}}\right)^{\frac{1}{\theta}}\right] .
$$

Note that $\lim _{n \rightarrow \infty} \frac{a_{n}}{\lambda_{n}}=0$ and $\sum_{n=1}^{\infty} \alpha_{n} \lambda_{n}=\infty$. Using Lemma 2.4, we obtain $x_{n} \rightarrow x^{*}$. This completes the proof.

If we put $g=0$ in (3.3), then this triple hierarchical variational inequality reduces to the variational inequality (3.18). Thus, the following is the direct consequence of Theorem 3.1.

Theorem 3.2 Let $C$ be a nonempty closed convex subset of a real Hilbert space H. Let $F: C \rightarrow H$ be a $k$-Lipschitzian and $\eta$-strongly monotone operator, and $S: C \rightarrow C$ be a nonexpansive mapping. Let $A: C \rightarrow H$ be an $\alpha$-inverse strongly monotone mapping and $\mathcal{T}=\left\{T_{n}\right\}$ be a sequence of nearly nonexpansive mappings from $C$ into itself with respect to a sequence $\left\{a_{n}\right\}$ such that $\sum_{n=1}^{\infty} \mathcal{D}_{B}\left(T_{n}, T_{n+1}\right)<\infty$ for all $B \in \mathcal{B}(C)$ and $F(\mathcal{T}) \cap \Omega(C, A) \neq \emptyset$ and let $T$ be a mapping from $C$ into itself defined by $T x=\lim _{n \rightarrow \infty} T_{n} x$ for all $x \in C$. Suppose that $F(T)=F(\mathcal{T}), 0<\mu<\frac{2 \eta}{k^{2}}$, and $0<\gamma \leq \tau$, where $\tau=1-\sqrt{1-\mu\left(2 \eta-\mu k^{2}\right)}$. Assume that $\Omega$, the set of solutions of the hierarchical variational inequality (3.1), is nonempty. Consider the sequence $\left\{x_{n}\right\}$ in $C$ for arbitrary $x_{1} \in C$, generated by the following iterative process:

$$
\left\{\begin{array}{l}
x_{1} \in C, \\
y_{n}=T_{n} P_{C}\left[x_{n}-t_{n} A x_{n}\right], \\
x_{n+1}=P_{C}\left[\lambda_{n}\left(1-\alpha_{n}\right) \gamma S x_{n}+\left(I-\lambda_{n} \mu F\right) y_{n}\right]
\end{array}\right.
$$

for all $n \in \mathbb{N}$, to be bounded and $\lim _{n \rightarrow \infty} \frac{\left\|x_{n}-P_{C}\left[x_{n}-t_{n} A x_{n}\right]\right\|}{\lambda_{n}}=0$, where $\left\{\alpha_{n}\right\},\left\{\lambda_{n}\right\}$, and $\left\{t_{n}\right\}$ are sequences mentioned in Theorem 3.1 satisfying all the conditions of Theorem 3.1. Then the sequence $\left\{x_{n}\right\}$ converges strongly to a unique solution $x^{*}$ of the variational inequality of 
finding $x^{*} \in \Omega$ such that

$$
\left\langle F x^{*}, x-x^{*}\right\rangle \geq 0, \quad \forall x \in \Omega .
$$

Take $T_{n}=T$ and $A=0$ in Theorem 3.1, we have the following.

Corollary 3.1 (Ceng et al. [11, Theorem 4.1]) Let $C$ be a nonempty closed convex subset of a real Hilbert space $H$. Let $F: C \rightarrow H$ be a $k$-Lipschitzian and $\eta$-strongly monotone operator, and $g: C \rightarrow H$ be a $\rho$-contraction mapping. Let $S$ and $T$ be nonexpansive mappings from $C$ into itself such that $F(T) \neq \emptyset$. Suppose that $0<\mu<\frac{2 \eta}{k^{2}}$ and $0<\gamma \leq \tau$, where $\tau=1-\sqrt{1-\mu\left(2 \eta-\mu k^{2}\right)}$. Assume that $\Omega$, the set of solutions of the hierarchical variational inequality of finding $z^{*} \in F(T)$ such that

$$
\left\langle(\mu F-\gamma S) z^{*}, z-z^{*}\right\rangle \geq 0, \quad \forall z \in F(T),
$$

is nonempty. Consider the sequence $\left\{x_{n}\right\}$ in C for arbitrary $x_{1} \in C$, generated by the following iterative process:

$$
\left\{\begin{array}{l}
x_{1} \in C, \\
x_{n+1}=P_{C}\left[\lambda_{n} \gamma\left(\alpha_{n} g\left(x_{n}\right)+\left(1-\alpha_{n}\right) S x_{n}\right)+\left(I-\lambda_{n} \mu F\right) T x_{n}\right]
\end{array}\right.
$$

for all $n \in \mathbb{N}$, where $\left\{\alpha_{n}\right\}$ and $\left\{\lambda_{n}\right\}$ are sequences in $(0,1)$ satisfying the conditions (i)-(ii) of Theorem 3.1. Suppose that $\lim _{n \rightarrow \infty} \frac{\lambda_{n}^{\frac{1}{\theta}}}{\alpha_{n}}=0$, and $\|x-T x\| \geq \bar{k}[d(x, F(T))]^{\theta}, \forall x \in C$, where $\bar{k}>0$ and $\theta>0$ are constants. Then the following hold:

(a) If the generated sequence $\left\{x_{n}\right\}$ is bounded, then the sequence $\left\{x_{n}\right\}$ converges strongly to the point $x^{*} \in F(T)$, where $x^{*}$ is the unique solution of the triple hierarchical variational inequality of finding $x^{*} \in \Omega$ such that

$$
\left\langle(\mu F-\gamma g) x^{*}, x-x^{*}\right\rangle \geq 0, \quad \forall x \in \Omega \text {. }
$$

(b) If the sequence $\left\{x_{n}\right\}$ in $C$ for arbitrary $x_{1} \in C$, generated by the following iterative process:

$$
\left\{\begin{array}{l}
x_{1} \in C \\
x_{n+1}=P_{C}\left[\lambda_{n}\left(1-\alpha_{n}\right) \gamma S x_{n}+\left(I-\lambda_{n} \mu F\right) T x_{n}\right]
\end{array}\right.
$$

for all $n \in \mathbb{N}$, is bounded, then the sequence $\left\{x_{n}\right\}$ converges strongly to the unique solution $x^{*}$ of the variational inequality of finding $x^{*} \in \Omega$ such that

$$
\left\langle F x^{*}, x-x^{*}\right\rangle \geq 0, \quad \forall x \in \Omega .
$$

We now derive the result of Yao et al. [10, Theorem 4.1] as a corollary.

Corollary 3.2 Let $C$ be a nonempty closed convex subset of a real Hilbert space $H$. Let $f: C \rightarrow H$ be a $\rho$-contraction mapping. Let $S$ and $T$ be nonexpansive mappings from $C$ into itself such that $F(T) \neq \emptyset$. Assume that $\Omega$, the set of solutions of the hierarchical variational inequality of finding $x^{*} \in F(T)$ such that

$$
\left\langle(I-S) x^{*}, x-x^{*}\right\rangle \geq 0, \quad \forall x \in F(T),
$$


is nonempty. Consider the sequence $\left\{x_{n}\right\}$ in $C$ for arbitrary $x_{1} \in C$, generated by the following iterative process:

$$
\left\{\begin{array}{l}
x_{1} \in C, \\
x_{n+1}=P_{C}\left[\lambda_{n}\left(\alpha_{n} f\left(x_{n}\right)+\left(1-\alpha_{n}\right) S x_{n}\right)+\left(1-\lambda_{n}\right) T x_{n}\right]
\end{array}\right.
$$

for all $n \in \mathbb{N}$, where $\left\{\alpha_{n}\right\}$ and $\left\{\lambda_{n}\right\}$ are sequences in $(0,1)$ satisfying the conditions (i)-(ii) of Theorem 3.1. Suppose that $\lim _{n \rightarrow \infty} \frac{\lambda_{n}^{\frac{1}{\theta}}}{\alpha_{n}}=0$ and $\|x-T x\| \geq \bar{k}[d(x, F(T))]^{\theta}, \forall x \in C$, where $\bar{k}>0$ and $\theta>0$ are constants. Then:

(a) If the generated sequence $\left\{x_{n}\right\}$ is bounded, then the sequence $\left\{x_{n}\right\}$ converges strongly to the point $x^{*} \in F(T)$, where $x^{*}$ is the unique solution of the variational inequality of finding $x^{*} \in \Omega$ such that

$$
\left\langle(I-f) x^{*}, x-x^{*}\right\rangle \geq 0, \quad \forall x \in \Omega .
$$

(b) If the sequence $\left\{x_{n}\right\}$ in $C$ for arbitrary $x_{1} \in C$, generated by the following iterative process:

$$
\left\{\begin{array}{l}
x_{1} \in C \\
x_{n+1}=P_{C}\left[\lambda_{n}\left(1-\alpha_{n}\right) S x_{n}+\left(1-\lambda_{n}\right) T x_{n}\right]
\end{array}\right.
$$

for all $n \in \mathbb{N}$, is bounded, then the sequence $\left\{x_{n}\right\}$ converges strongly to a minimum norm solution of the hierarchical variational inequality (3.19).

Again, we derive the following result as a corollary for $S$ and $T$ being two nonexpansive mappings.

Corollary 3.3 Let $C$ be a nonempty closed convex subset of a real Hilbert space H. Let $F: C \rightarrow H$ be a k-Lipschitzian and $\eta$-strongly monotone operator, and $g: C \rightarrow H$ be a $\rho$-contraction mapping. Let $S$ and $T$ be nonexpansive mappings from $C$ into itself and $A$ : $C \rightarrow H$ be an $\alpha$-inverse strongly monotone mapping such that $F(T) \cap \Omega(C, A) \neq \emptyset$. Suppose that $0<\mu<\frac{2 \eta}{k^{2}}$ and $0<\gamma \leq \tau$, where $\tau=1-\sqrt{1-\mu\left(2 \eta-\mu k^{2}\right)}$. Assume that $\Omega$, the set of solutions of the hierarchical variational inequality of finding $z^{*} \in F(T) \cap \Omega(C, A)$ such that

$$
\left\langle(\mu F-\gamma S) z^{*}, z-z^{*}\right\rangle \geq 0, \quad \forall z \in F(T) \cap \Omega(C, A),
$$

is nonempty. Consider the sequence $\left\{x_{n}\right\}$ in $C$ for arbitrary $x_{1} \in C$, generated by the following iterative process:

$$
\left\{\begin{array}{l}
x_{1} \in C, \\
y_{n}=T P_{C}\left[x_{n}-t_{n} A x_{n}\right], \\
x_{n+1}=P_{C}\left[\lambda_{n} \gamma\left(\alpha_{n} g\left(x_{n}\right)+\left(1-\alpha_{n}\right) S x_{n}\right)+\left(I-\lambda_{n} \mu F\right) y_{n}\right]
\end{array}\right.
$$

for all $n \in \mathbb{N}$, where $\left\{\alpha_{n}\right\},\left\{\lambda_{n}\right\}$ are sequences in $(0,1)$ and $\left\{t_{n}\right\}$ is a sequence in $[a, b]$ (for some $a, b$ with $0<a<b<2 \alpha$ ) satisfying the conditions (i)-(ii) of Theorem 3.1. Suppose that 
$\lim _{n \rightarrow \infty} \frac{\lambda_{n}^{\frac{1}{\theta}}}{\alpha_{n}}=0, \sum_{n=1}^{\infty}\left|t_{n+1}-t_{n}\right|<\infty, \lim _{n \rightarrow \infty} \frac{\left|t_{n}-t_{n-1}\right|}{\alpha_{n} \lambda_{n}^{2}}=0$ and $\|x-T x\| \geq \bar{k}[d(x, F(T) \cap$ $\Omega(C, A))]^{\theta}, \forall x \in C$, where $\bar{k}>0$ and $\theta>0$ are constants. Then the following hold:

(a) If the generated sequence $\left\{x_{n}\right\}$ is bounded and $\lim _{n \rightarrow \infty} \frac{\left\|x_{n}-P_{C}\left[x_{n}-t_{n} A x_{n}\right]\right\|}{\lambda_{n}}=0$, then the sequence $\left\{x_{n}\right\}$ converges strongly to the point $x^{*} \in F(T) \cap \Omega(C, A)$, where $x^{*}$ is the unique solution of the triple hierarchical variational inequality of finding $x^{*} \in \Omega$ such that

$$
\left\langle(\mu F-\gamma g) x^{*}, x-x^{*}\right\rangle \geq 0, \quad \forall x \in \Omega
$$

(b) If the sequence $\left\{x_{n}\right\}$ in $C$ for arbitrary $x_{1} \in C$, generated by the following iterative process:

$$
\left\{\begin{array}{l}
x_{1} \in C \\
y_{n}=T P_{C}\left[x_{n}-t_{n} A x_{n}\right] \\
x_{n+1}=P_{C}\left[\lambda_{n}\left(1-\alpha_{n}\right) \gamma S x_{n}+\left(I-\lambda_{n} \mu F\right) y_{n}\right]
\end{array}\right.
$$

for all $n \in \mathbb{N}$, is bounded and $\lim _{n \rightarrow \infty} \frac{\left\|x_{n}-P_{C}\left[x_{n}-t_{n} A x_{n}\right]\right\|}{\lambda_{n}}=0$, then the sequence $\left\{x_{n}\right\}$ converges strongly to the unique solution $x^{*}$ of the variational inequality of finding $x^{*} \in \Omega$ such that

$$
\left\langle F x^{*}, x-x^{*}\right\rangle \geq 0, \quad \forall x \in \Omega \text {. }
$$

\section{Applications}

In this section, we present two applications of Theorem 3.1. The first application is concerned with the image recovery problem which is equivalent to finding a common fixed point of finitely many nonexpansive self mappings. The first application improves a number of results related to this context. The second application deals with a strictly pseudocontractive mapping.

Theorem 4.1 Let $C$ be a nonempty closed convex subset of a real Hilbert space H. Let $F: C \rightarrow H$ be a k-Lipschitzian and $\eta$-strongly monotone operator, and $g: C \rightarrow H$ be a $\rho$-contraction mapping. Let $S: C \rightarrow C$ be a nonexpansive mapping and $A: C \rightarrow H$ be an $\alpha$-inverse strongly monotone mapping. Let $t_{1}, t_{2}, t_{3}, \ldots, t_{N}>0$ such that $\sum_{i=1}^{N} t_{i}=1$. Let $T_{1}, T_{2}, T_{3}, \ldots, T_{N}: C \rightarrow C$ be nonexpansive mappings such that $\bigcap_{i=1}^{N} F\left(T_{i}\right) \cap \Omega(C, A) \neq \emptyset$ and assume that $T=\sum_{i=1}^{N} t_{i} T_{i}$. Suppose that $0<\mu<\frac{2 \eta}{k^{2}}$ and $0<\gamma \leq \tau$, where $\tau=1-$ $\sqrt{1-\mu\left(2 \eta-\mu k^{2}\right)}$. Assume that $\Omega$, the set of solutions of the hierarchical variational inequality of finding $z^{*} \in \bigcap_{i=1}^{N} F\left(T_{i}\right) \cap \Omega(C, A)$ such that

$$
\left\langle(\mu F-\gamma S) z^{*}, z-z^{*}\right\rangle \geq 0, \quad \forall z \in \bigcap_{i=1}^{N} F\left(T_{i}\right) \cap \Omega(C, A),
$$

is nonempty. Consider the sequence $\left\{x_{n}\right\}$ in $C$ for arbitrary $x_{1} \in C$, generated by the following iterative process:

$$
\left\{\begin{array}{l}
x_{1} \in C, \\
y_{n}=\sum_{i=1}^{N} t_{i} T_{i} P_{C}\left[x_{n}-t_{n} A x_{n}\right], \\
x_{n+1}=P_{C}\left[\lambda_{n} \gamma\left(\alpha_{n} g\left(x_{n}\right)+\left(1-\alpha_{n}\right) S x_{n}\right)+\left(I-\lambda_{n} \mu F\right) y_{n}\right]
\end{array}\right.
$$


for all $n \in \mathbb{N}$, where $\left\{\alpha_{n}\right\},\left\{\lambda_{n}\right\}$ are sequences in $(0,1)$ and $\left\{t_{n}\right\}$ is a sequence in $[a, b]$ (for some $a, b$ with $0<a<b<2 \alpha$ ) satisfying all the conditions of Corollary 3.3. Then the following hold:

(a) If the generated sequence $\left\{x_{n}\right\}$ is bounded and $\lim _{n \rightarrow \infty} \frac{\left\|x_{n}-P_{C}\left[x_{n}-t_{n} A x_{n}\right]\right\|}{\lambda_{n}}=0$, then the sequence $\left\{x_{n}\right\}$ converges strongly to the point $x^{*} \in \bigcap_{i=1}^{N} F\left(T_{i}\right) \cap \Omega(C, A)$, where $x^{*}$ is the unique solution of the triple hierarchical variational inequality of finding $x^{*} \in \Omega$ such that

$$
\left\langle(\mu F-\gamma g) x^{*}, x-x^{*}\right\rangle \geq 0, \quad \forall x \in \Omega \text {. }
$$

(b) If the sequence $\left\{x_{n}\right\}$ in $C$ for arbitrary $x_{1} \in C$, generated by the following iterative process:

$$
\left\{\begin{array}{l}
x_{1} \in C \\
y_{n}=\sum_{i=1}^{N} t_{i} T_{i} P_{C}\left[x_{n}-t_{n} A x_{n}\right] \\
x_{n+1}=P_{C}\left[\lambda_{n}\left(1-\alpha_{n}\right) \gamma S x_{n}+\left(I-\lambda_{n} \mu F\right) y_{n}\right]
\end{array}\right.
$$

for all $n \in \mathbb{N}$, is bounded and $\lim _{n \rightarrow \infty} \frac{\left\|x_{n}-P_{C}\left[x_{n}-t_{n} A x_{n}\right]\right\|}{\lambda_{n}}=0$, then the sequence $\left\{x_{n}\right\}$ converges strongly to the unique solution $x^{*}$ of the variational inequality of finding $x^{*} \in \Omega$ such that

$$
\left\langle F x^{*}, x-x^{*}\right\rangle \geq 0, \quad \forall x \in \Omega
$$

Proof Lemma 2.5 implies that $T$ is nonexpansive from $C$ into itself and $F(T)=\bigcap_{i=1}^{N} F\left(T_{i}\right)$. Hence, the result follows from Corollary 3.3.

Theorem 4.2 Let $C$ be a nonempty closed convex subset of a real Hilbert space $H$. Let $F: C \rightarrow H$ be a k-Lipschitzian and $\eta$-strongly monotone operator, and $g: C \rightarrow H$ be a $\rho$-contraction mapping. Let $S: C \rightarrow C$ be a nonexpansive mapping. Let $U: C \rightarrow C$ be a $\lambda$-strictly pseudocontractive mapping and $\mathcal{T}=\left\{T_{n}\right\}$ be a sequence of nearly nonexpansive mappings from $C$ into itself with respect to a sequence $\left\{a_{n}\right\}$ such that $\sum_{n=1}^{\infty} \mathcal{D}_{B}\left(T_{n}, T_{n+1}\right)<\infty$ for all $B \in \mathcal{B}(C)$ and $F(\mathcal{T}) \cap F(U) \neq \emptyset$ and let $T$ be a mapping from $C$ into itself defined by $T x=\lim _{n \rightarrow \infty} T_{n} x$ for all $x \in C$. Suppose that $F(T)=F(\mathcal{T}), 0<\mu<\frac{2 \eta}{k^{2}}$, and $0<\gamma \leq \tau$, where $\tau=1-\sqrt{1-\mu\left(2 \eta-\mu k^{2}\right)}$. Assume that $\Omega$, the set of solutions of the hierarchical variational inequality of finding $z^{*} \in F(\mathcal{T}) \cap F(U)$ such that

$$
\left\langle(\mu F-\gamma S) z^{*}, z-z^{*}\right\rangle \geq 0, \quad \forall z \in F(\mathcal{T}) \cap F(U)
$$

is nonempty. Consider the sequence $\left\{x_{n}\right\}$ in $C$ for arbitrary $x_{1} \in C$, generated by the following iterative process:

$$
\left\{\begin{array}{l}
x_{1} \in C, \\
y_{n}=T_{n}\left[\left(1-t_{n}\right) x_{n}+t_{n} U x_{n}\right], \\
x_{n+1}=P_{C}\left[\lambda_{n} \gamma\left(\alpha_{n} g\left(x_{n}\right)+\left(1-\alpha_{n}\right) S x_{n}\right)+\left(I-\lambda_{n} \mu F\right) y_{n}\right]
\end{array}\right.
$$


for all $n \in \mathbb{N}$, where $\left\{\alpha_{n}\right\},\left\{\lambda_{n}\right\}$ are sequences in $(0,1)$ and $\left\{t_{n}\right\}$ is a sequence in $[a, b]$ (for some $a, b$ with $0<a<b<1-\lambda$ ) satisfying the conditions (i)-(iv) of Theorem 3.1. Suppose that $\left\|x-T_{n} x\right\| \geq \bar{k}[d(x, F(\mathcal{T}) \cap F(U))]^{\theta}, \forall x \in C$ and $n \in \mathbb{N}$, where $\bar{k}>0$ and $\theta>0$ are constants. Then the following hold:

(a) If the generated sequence $\left\{x_{n}\right\}$ is bounded and $\lim _{n \rightarrow \infty} \frac{\left\|x_{n}-U x_{n}\right\|}{\lambda_{n}}=0$, then the sequence $\left\{x_{n}\right\}$ converges strongly to the point $x^{*} \in F(\mathcal{T}) \cap F(U)$, where $x^{*}$ is the unique solution of the triple hierarchical variational inequality of finding $x^{*} \in \Omega$ such that

$$
\left\langle(\mu F-\gamma g) x^{*}, x-x^{*}\right\rangle \geq 0, \quad \forall x \in \Omega .
$$

(b) If the sequence $\left\{x_{n}\right\}$ in $C$ for arbitrary $x_{1} \in C$, generated by the following iterative process:

$$
\left\{\begin{array}{l}
x_{1} \in C, \\
y_{n}=T_{n}\left[\left(1-t_{n}\right) x_{n}+t_{n} U x_{n}\right], \\
x_{n+1}=P_{C}\left[\lambda_{n}\left(1-\alpha_{n}\right) \gamma S x_{n}+\left(I-\lambda_{n} \mu F\right) y_{n}\right]
\end{array}\right.
$$

for all $n \in \mathbb{N}$, is bounded and $\lim _{n \rightarrow \infty} \frac{\left\|x_{n}-U x_{n}\right\|}{\lambda_{n}}=0$, then the sequence $\left\{x_{n}\right\}$ converges strongly to the unique solution $x^{*}$ of the variational inequality of finding $x^{*} \in \Omega$ such that

$$
\left\langle F x^{*}, x-x^{*}\right\rangle \geq 0, \quad \forall x \in \Omega
$$

Proof Put $A=I-U$ in Theorem 3.1, then $A$ is $\frac{(1-\lambda)}{2}$-inverse strongly monotone. We also have $F(U)=\Omega(C, A)$ and $P_{C}\left(x_{n}-t_{n} A x_{n}\right)=\left(1-t_{n}\right) x_{n}+t_{n} U x_{n}$. Therefore, the conclusion follows from Theorem 3.1 and Theorem 3.2.

\section{Numerical example}

In this section, we discuss the following example which shows the effectiveness and convergence of iteratively generated sequence $\left\{x_{n}\right\}$ by the considered scheme (3.2) of Theorem 3.1.

Example 5.1 Let $H=\mathbb{R}$ and $C=[0,1]$. Let $A, S$, and $T$ be mappings defined by $A(x)=$ $2 x-1, S(x)=x$, and $T(x)=1-x$ for all $x \in C$.

Let $F, g: C \rightarrow H$ be mappings defined by $F(x)=2 x$ and $g(x)=\frac{x}{2}+1$ for all $x \in C$. Define $\left\{t_{n}\right\},\left\{\alpha_{n}\right\}$, and $\left\{\lambda_{n}\right\}$ in $(0,1)$ by $t_{n}=\frac{1}{2}, \alpha_{n}=\frac{1}{(n+2)^{p}}$, and $\lambda_{n}=\frac{1}{(n+2)^{q}}$, where $0<p+2 q<1$. It is clear that $S$ and $T$ are nonexpansive self mappings, and $A$ is 2 -inverse strongly monotone. Note $F$ is a 2-Lipschitzian and 2-strongly monotone, and $g$ is a $\frac{1}{2}$-contraction mapping. Here, $k=2, \eta=2$, and $\rho=\frac{1}{2}$. We take $\mu=\frac{1}{4}, \gamma=\tau=1-\sqrt{1-\mu\left(2 \eta-\mu k^{2}\right)}=\frac{1}{2}, p=\frac{1}{2}$, $q=\frac{1}{6}$, and $\theta=\frac{1}{4}$. Note that $0<\mu<\frac{2 \eta}{k^{2}}$. Observe that $T_{n}=T$ with $a_{n}=0$ for all $n \in \mathbb{N}$ and $F(\mathcal{T}) \cap \Omega(C, A)=\left\{\frac{1}{2}\right\}$. The iterative algorithm (3.2) can be written as

$$
x_{n+1}=P_{C}\left(u_{n}\right) \quad \text { for all } n \in \mathbb{N},
$$

where

$$
\begin{aligned}
& u_{n}=\lambda_{n} \gamma\left(\alpha_{n} g\left(x_{n}\right)+\left(1-\alpha_{n}\right) S x_{n}\right)+\left(I-\lambda_{n} \mu F\right) y_{n}, \\
& y_{n}=T_{n} z_{n}, \quad z_{n}=P_{C}\left(x_{n}-t_{n} A x_{n}\right) .
\end{aligned}
$$


We observe that

$$
\begin{aligned}
& z_{n}=P_{C}\left(x_{n}-t_{n} A x_{n}\right)=P_{C}\left(x_{n}-\frac{1}{2}\left(2 x_{n}-1\right)\right)=P_{C}\left(\frac{1}{2}\right)=\frac{1}{2}, \\
& y_{n}=T_{n} z_{n}=T(1 / 2)=1 / 2
\end{aligned}
$$

and

$$
\begin{aligned}
u_{n} & =\lambda_{n} \gamma\left(\alpha_{n} g\left(x_{n}\right)+\left(1-\alpha_{n}\right) S x_{n}\right)+\left(I-\lambda_{n} \mu F\right) y_{n} \\
& =\frac{\lambda_{n}}{2}\left(\alpha_{n}\left(\frac{x_{n}}{2}+1\right)+\left(1-\alpha_{n}\right) x_{n}\right)+\frac{1}{2}-\frac{\lambda_{n}}{4} F\left(\frac{1}{2}\right) \\
& =\frac{\lambda_{n}}{2}\left(\alpha_{n}+\left(1-\frac{\alpha_{n}}{2}\right) x_{n}\right)+\frac{1}{2}-\frac{\lambda_{n}}{4} .
\end{aligned}
$$

Let $x_{1} \in C$. For $n=1$, we have

$$
\begin{aligned}
u_{1} & =\frac{\lambda_{1}}{2}\left(\alpha_{1}+\left(1-\frac{\alpha_{1}}{2}\right) x_{1}\right)+\frac{1}{2}-\frac{\lambda_{1}}{4} \\
& \leq \frac{\lambda_{1}}{2}\left(\alpha_{1}+\left(1-\frac{\alpha_{1}}{2}\right)\right)+\frac{1}{2}-\frac{\lambda_{1}}{4} \\
& =\frac{1}{2(3)^{\frac{1}{6}}}\left(\frac{1}{2(3)^{\frac{1}{2}}}+1\right)+\frac{1}{2}-\frac{1}{4(3)^{\frac{1}{6}}} \\
& =\frac{1}{4(3)^{\frac{1}{6}}}+\frac{1}{4(3)^{\frac{2}{3}}}+\frac{1}{2}=0.8284<1 .
\end{aligned}
$$

Thus, $u_{1} \in C$.

Next we show that $x_{n} \in C$ for all $n \in \mathbb{N}$. Note $u_{1} \in C$. Suppose that $u_{k} \in C$ for some $k \in \mathbb{N}$. Now, for $n=k+1$, we have

$$
\begin{aligned}
u_{k+1} & =\frac{\lambda_{k+1}}{2}\left(\alpha_{k+1}+\left(1-\frac{\alpha_{k+1}}{2}\right) x_{k+1}\right)+\frac{1}{2}-\frac{\lambda_{k+1}}{4} \\
& =\frac{\lambda_{k+1}}{2}\left(\alpha_{k+1}+\left(1-\frac{\alpha_{k+1}}{2}\right) P_{C} u_{k}\right)+\frac{1}{2}-\frac{\lambda_{k+1}}{4} \\
& =\frac{\lambda_{k+1}}{2}\left(\alpha_{k+1}+\left(1-\frac{\alpha_{k+1}}{2}\right) u_{k}\right)+\frac{1}{2}-\frac{\lambda_{k+1}}{4} \\
& \leq \frac{\lambda_{k+1}}{2}\left(\alpha_{k+1}+\left(1-\frac{\alpha_{k+1}}{2}\right)\right)+\frac{1}{2}-\frac{\lambda_{k+1}}{4} \\
& =\frac{\lambda_{k+1}}{2}\left(\frac{\alpha_{k+1}}{2}+1\right)+\frac{1}{2}-\frac{\lambda_{k+1}}{4} \\
& =\frac{\lambda_{k+1}}{4}+\frac{1}{2}+\frac{\lambda_{k+1} \alpha_{k+1}}{4} \\
& <\frac{1}{4}+\frac{1}{4}+\frac{1}{2}=1
\end{aligned}
$$

Thus, by mathematical induction, we get $u_{n} \in C$ for all $n \in \mathbb{N}$. Therefore, $x_{n} \in C$ for all $n \in \mathbb{N}$. It can be seen from Table 1 and Figure 1 that $\left\{\frac{\left|x_{n}-z_{n}\right|}{\lambda_{n}}\right\}$ converges to 0 (see also (5.3)). 
Table 1 The numerical values of $\frac{\left|x_{n}-z_{n}\right|}{\lambda_{n}}$ up to $n=10,000$

\begin{tabular}{rlllll}
\hline $\boldsymbol{n}$ & $\frac{\left|\boldsymbol{x}_{\boldsymbol{n}}-\boldsymbol{z}_{\boldsymbol{n}}\right|}{\lambda_{\boldsymbol{n}}}$ & $\boldsymbol{n}$ & $\frac{\left|\boldsymbol{x}_{\boldsymbol{n}}-\boldsymbol{z}_{\boldsymbol{n}}\right|}{\lambda_{\boldsymbol{n}}}$ & $\boldsymbol{n}$ & $\frac{\left|\boldsymbol{x}_{\boldsymbol{n}}-\boldsymbol{z}_{\boldsymbol{n}}\right|}{\lambda \boldsymbol{n}_{\boldsymbol{n}}}$ \\
\hline 1 & 0.600468477588001 & 3,400 & 0.007373276312992 & 6,800 & 0.005133489777493 \\
200 & 0.033090574752038 & 3,600 & 0.007155873637358 & 7,000 & 0.005056543877199 \\
400 & 0.022842404623134 & 3,800 & 0.006956215006767 & 7,200 & 0.004982885604091 \\
600 & 0.018408162394866 & 4,000 & 0.006772024658859 & 7,400 & 0.004912288613870 \\
800 & 0.015801751117225 & 4,200 & 0.006601414499865 & 7,600 & 0.0048445477738281 \\
1,000 & 0.014040689141910 & 4,400 & 0.006442803256853 & 7,800 & 0.004779476507962 \\
1,200 & 0.012750658965951 & 4,600 & 0.006294855306963 & 8,000 & 0.004716905020154 \\
1,400 & 0.011754246690473 & 4,800 & 0.006156433766950 & 8,200 & 0.004656678095976 \\
1,600 & 0.010955215843824 & 5,000 & 0.006026564076254 & 8,400 & 0.004598653681922 \\
1,800 & 0.010296300001474 & 5,200 & 0.005904405409570 & 8,600 & 0.004542701458279 \\
2,000 & 0.009741032549748 & 5,400 & 0.005789228005585 & 8,800 & 0.004488701623540 \\
2,200 & 0.009264955188978 & 5,600 & 0.005680395018282 & 9,000 & 0.004436543829128 \\
2,400 & 0.008850976030794 & 5,800 & 0.005577347862578 & 9,200 & 0.004386126242951 \\
2,600 & 0.008486746121476 & 6,000 & 0.005479594286560 & 9,400 & 0.004337354723797 \\
2,800 & 0.008163093477629 & 6,200 & 0.005386698590768 & 9,600 & 0.004290142091386 \\
3,000 & 0.007873045437275 & 6,400 & 0.005298273552540 & 9,800 & 0.004244407479276 \\
3,200 & 0.007611194925153 & 6,600 & 0.005213973715158 & 10,000 & 0.004200075759726 \\
\hline & & & & &
\end{tabular}

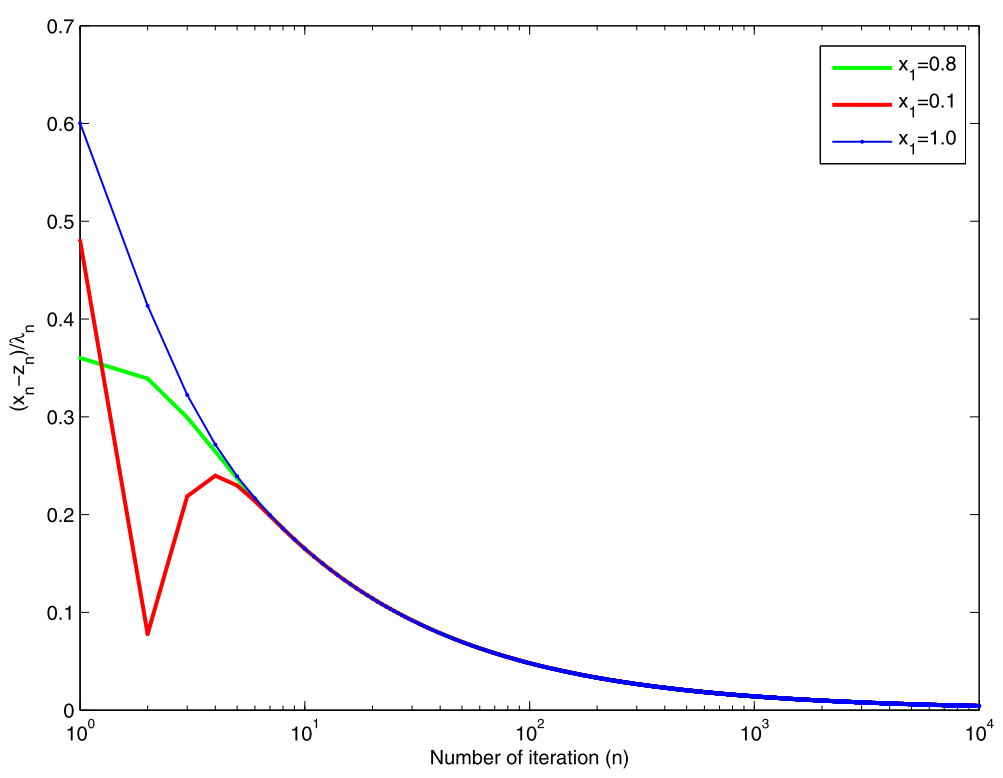

Figure 1 Convergence of sequence $\left\{\frac{\left|x_{n}-z_{n}\right|}{\lambda_{n}}\right\}$.

Thus, all the assumptions of Theorem 3.1 are satisfied. Therefore, the iteratively generated sequence $\left\{x_{n}\right\}$ defined by (3.2) converges strongly to $\left\{\frac{1}{2}\right\}$, which is also the unique solution of the triple hierarchical variational inequality (3.3).

The numerical values of $x_{n}$ up to $n=10,000$ have been calculated in Table 2 and convergence of sequence $\left\{x_{n}\right\}$ is given in Figure 2. Finally, mathematically, we show that $x_{n} \rightarrow \frac{1}{2}$ and $\frac{\left|x_{n}-z_{n}\right|}{\lambda_{n}} \rightarrow 0$ as $n \rightarrow \infty$. Note

$$
\begin{aligned}
x_{n+1} & =P_{C}\left(u_{n}\right)=u_{n}=\frac{\lambda_{n}}{2}\left(\alpha_{n}+\left(1-\frac{\alpha_{n}}{2}\right) x_{n}\right)+\frac{1}{2}-\frac{\lambda_{n}}{4} \\
& \leq \frac{\lambda_{n}}{2}\left(\alpha_{n}+\left(1-\frac{\alpha_{n}}{2}\right)\right)+\frac{1}{2}-\frac{\lambda_{n}}{4}=\frac{\alpha_{n} \lambda_{n}}{4}+\frac{\lambda_{n}}{4}+\frac{1}{2},
\end{aligned}
$$


Table 2 The numerical values of $x_{n}$ up to $n=10,000$

\begin{tabular}{rlllll}
\hline $\boldsymbol{n}$ & $\boldsymbol{x}_{\boldsymbol{n}}$ & $\boldsymbol{n}$ & $\boldsymbol{x}_{\boldsymbol{n}}$ & $\boldsymbol{n}$ & $\boldsymbol{x}_{\boldsymbol{n}}$ \\
\hline 1 & 0.000000000000000 & 3,400 & 0.501901245173150 & 6,800 & 0.501179342447758 \\
200 & 0.513660892766142 & 3,600 & 0.501827701962605 & 7,000 & 0.501156068130334 \\
400 & 0.508408221515529 & 3,800 & 0.501760776817138 & 7,200 & 0.501133892973359 \\
600 & 0.506334966973673 & 4,000 & 0.501699569844454 & 7,400 & 0.501112736570979 \\
800 & 0.505184133249873 & 4,200 & 0.501643340889305 & 7,600 & 0.501092526371813 \\
1,000 & 0.504438577466294 & 4,400 & 0.501591475155260 & 7,800 & 0.501073196728994 \\
1,200 & 0.503910346287644 & 4,600 & 0.501543457407883 & 8,000 & 0.501054688086088 \\
1,400 & 0.503513474443515 & 4,800 & 0.501498852346845 & 8,200 & 0.501036946276588 \\
1,600 & 0.503202657672226 & 5,000 & 0.501457289482619 & 8,400 & 0.501019921918822 \\
1,800 & 0.502951585650275 & 5,200 & 0.501418451349507 & 8,600 & 0.501003569891334 \\
2,000 & 0.502743853895386 & 5,400 & 0.501382064222004 & 8,800 & 0.500987848876446 \\
2,200 & 0.502568662846337 & 5,600 & 0.501347890731946 & 9,000 & 0.500972720961835 \\
2,400 & 0.502418590433880 & 5,800 & 0.501315723944806 & 9,200 & 0.500958151291612 \\
2,600 & 0.502288354629419 & 6,000 & 0.501285382567478 & 9,400 & 0.500944107759859 \\
2,800 & 0.502174086142696 & 6,200 & 0.501256707041723 & 9,600 & 0.500930560740660 \\
3,000 & 0.502072880709147 & 6,400 & 0.501229556336889 & 9,800 & 0.500917482849616 \\
3,200 & 0.501982512601766 & 6,600 & 0.501203805299252 & 10,000 & 0.500904848732640 \\
\hline & & & & &
\end{tabular}

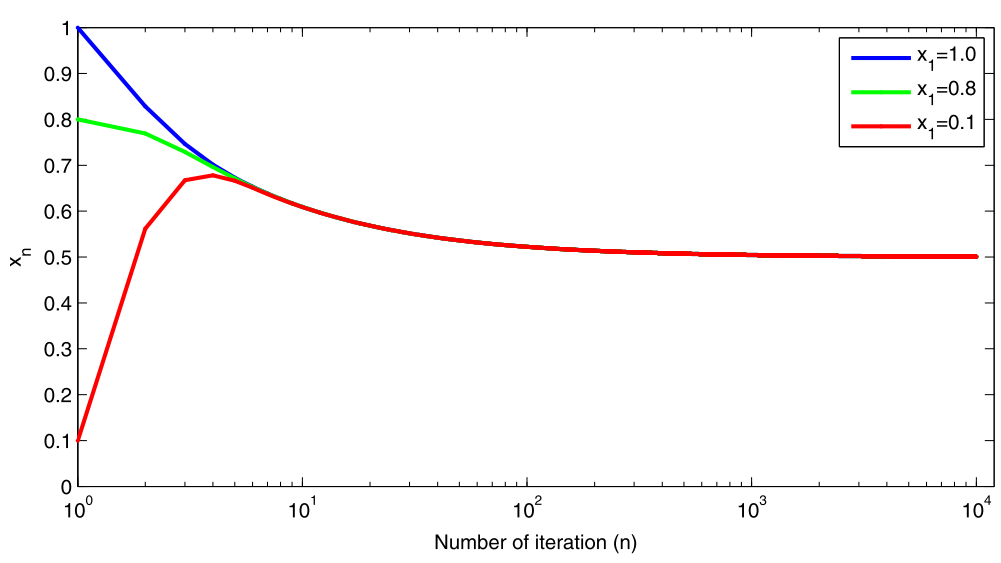

Figure 2 Convergence of the sequence $\left\{x_{n}\right\}$.

which implies that

$$
\left|x_{n+1}-\frac{1}{2}\right| \leq \frac{\alpha_{n} \lambda_{n}}{4}+\frac{\lambda_{n}}{4} \rightarrow 0 \quad \text { as } n \rightarrow \infty \text {. }
$$

Therefore, from (5.2) we get $x_{n} \rightarrow \frac{1}{2}$ as $n \rightarrow \infty$.

From (5.1), we have

$$
x_{n+1}-\frac{1}{2}=\frac{\lambda_{n}}{2}\left[\alpha_{n}+x_{n}-\frac{1}{2}-\frac{\alpha_{n} x_{n}}{2}\right] \text {, }
$$

which implies that

$$
\frac{\left|x_{n+1}-\frac{1}{2}\right|}{\lambda_{n+1}}=\frac{\lambda_{n}}{2 \lambda_{n+1}}\left|\alpha_{n}+x_{n}-\frac{1}{2}-\frac{\alpha_{n} x_{n}}{2}\right| \text {. }
$$


We have $\alpha_{n} \rightarrow 0$ and $\lambda_{n} \rightarrow 0$ as $n \rightarrow \infty$. Thus, we obtain

$$
\frac{\left|x_{n+1}-z_{n}\right|}{\lambda_{n+1}}=\frac{\lambda_{n}}{2 \lambda_{n+1}}\left|\alpha_{n}+x_{n}-\frac{1}{2}-\frac{\alpha_{n} x_{n}}{2}\right| \rightarrow 0 \quad \text { as } n \rightarrow \infty
$$

\section{Competing interests}

The authors declare that they have no competing interests.

\section{Authors' contributions}

All authors read and approved the final manuscript.

\section{Author details}

${ }^{1}$ Department of Mathematics, Banaras Hindu University, Varanasi, 221005, India. ${ }^{2}$ Department of Mathematics and RINS, Gyeongsang National University, Jinju, 660-701, Korea.

\section{Acknowledgements}

The authors would like to thank the editor and referees for the useful comments and suggestions.

\section{Received: 12 August 2014 Accepted: 1 December 2014 Published: 23 Dec 2014}

\section{References}

1. Stampacchia, G: Formes bilineaires coercivities sur les ensembles convexes. C. R. Acad. Sci. Paris 258, $4413-4416$ (1964)

2. Kazmi, KR, Ahmad, N, Rizvi, SH: System of implicit nonconvex variational inequality problems: a projection method approach. J. Nonlinear Sci. Appl. 6, 170-180 (2013)

3. Noor, MA: Some developments in general variational inequalities. Appl. Math. Comput. 152, 199-277 (2004). doi:10.1016/S0096-3003(03)00558-7

4. Cianciaruso, F, Marino, G, Muglia, L, Yao, Y: On a two-step algorithm for hierarchical fixed point problems and variational inequalities. J. Inequal. Appl. 2009, Article ID 208692 (2009). doi:10.1155/2009/208692

5. Mainge, PE, Moudafi, A: Strong convergence of an iterative method for hierarchical fixed point problems. Pac J. Optim. 3, 529-538 (2007)

6. Moudafi, A, Mainge, PE: Towards viscosity approximations of hierarchical fixed points problems. Fixed Point Theory Appl. 2006, Article ID 95453 (2006). doi:10.1155/FPTA/2006/95453

7. Sahu, DR, Kang, SM, Sagar, V: Iterative methods for hierarchical common fixed point problems and variational inequalities. Fixed Point Theory Appl. 2013, 299 (2013). doi:10.1186/1687-1812-2013-299

8. liduka, $\mathrm{H}$ : Strong convergence for an iterative method for the triple hierarchical constrained optimization problem. Nonlinear Anal. 71, e1292-e1297 (2009). doi:10.1016/j.na.2009.01.133

9. Iiduka, $\mathrm{H}$ : Iterative algorithm for solving triple hierarchical constrained optimization problem. J. Optim. Theory Appl. 148, 580-592 (2011). doi:10.1007/s10957-010-9769-z

10. Yao, Y, Chen, $R, X u, H K$ : Schemes for finding minimum-norm solutions of variational inequalities. Nonlinear Anal. 72, 3447-3456 (2010). doi:10.1016/j.na.2009.12.029

11. Ceng, LC, Ansari, QH, Yao, JC: Iterative methods for triple hierarchical variational inequalities in Hilbert spaces. J. Optim. Theory Appl. 151, 489-512 (2011). doi:10.1007/s10957-011-9882-7

12. Ceng, LC, Ansari, QH, Yao, JC: Relaxed hybrid steepest-descent methods with variable parameters for triple-hierarchical variational inequalities. Appl. Anal. 91, 1793-1810 (2012). doi:10.1080/00036811.2011.614602

13. Ansari, QH, Ceng, LC, Gupta, H: Triple hierarchical variational inequalities. In: Ansari, QH (ed.) Nonlinear Analysis: Approximation Theory, Optimization and Applications, pp. 231-280. Springer, New York (2014)

14. Agarwal, RP, O’Regan, D, Sahu, DR: Fixed Point Theory for Lipschitzian-Type Mappings with Applications. Topological Fixed Point Theory and Its Applications. Springer, New York (2009)

15. Sahu, DR: Fixed points of demicontinuous nearly Lipschitzian mappings in Banach spaces. Comment. Math. Univ Carol. 46, 653-666 (2005)

16. Sahu, DR, Wong, NC, Yao, JC: A generalized hybrid steepest-descent method for variational inequalities in Banach spaces. Fixed Point Theory Appl. 2011, Article ID 754702 (2011). doi:10.1155/2011/754702

17. Goebel, K, Kirk, WA: Topics on Metric Fixed Point Theory. Cambridge University Press, Cambridge (1990)

18. Yamada, I: The hybrid steepest descent method for the variational inequality problem over the intersection of fixed point sets of nonexpansive mappings. In: Butnariu, D, Censor, Y, Reich, S (eds.) Inherently Parallel Algorithms in Feasibility and Optimization and Their Applications (Haifa, 2000). Stud. Comput. Math., vol. 8, pp. 473-504. North-Holland, Amsterdam (2001)

19. $\mathrm{Xu}, \mathrm{HK}, \mathrm{Kim}, \mathrm{TH}$ : Convergence of hybrid steepest-descent methods for variational inequalities. J. Optim. Theory Appl. 119, 185-201 (2003). doi:10.1023/B:JOTA.0000005048.79379.b6

20. Wong, NC, Sahu, DR, Yao, JC: Solving variational inequalities involving nonexpansive type mappings. Nonlinear Anal. 69, 4732-4753 (2008). doi:10.1016/j.na.2007.11.025

21. Takahashi, W, Toyoda, M: Weak convergence theorems for nonexpansive mappings and monotone mappings. J. Optim. Theory Appl. 118, 417-428 (2003). doi:10.1023/A:1025407607560

22. Kinderlehrer, D, Stampacchia, G: An Introduction to Variational Inequalities and Their Applications. Academic Press, New York (1980) 
10.1186/1687-1812-2014-244

Cite this article as: Sahu et al.: Iterative methods for triple hierarchical variational inequalities and common fixed point problems. Fixed Point Theory and Applications 2014, 2014:244

Submit your manuscript to a SpringerOpen ${ }^{\circ}$ journal and benefit from:

- Convenient online submission

- Rigorous peer review

- Immediate publication on acceptance

- Open access: articles freely available online

- High visibility within the field

- Retaining the copyright to your article

Submit your next manuscript at $\gg$ springeropen.com 